\title{
High Frequency Scattering by a Classically Invisible Body
}

\author{
E.Lakshtanov* $^{*} \quad$ B.D.Sleeman ${ }^{\dagger} \quad$ B. Vainberg $^{\ddagger}$
}

April 18, 2022

\begin{abstract}
We consider a polyhedron with zero classical resistance, i.e., a polyhedron invisible to an observer viewing only the paths of geometrical optics rays. The corresponding problem of scattering of plane waves by the polyhedron is studied. The quasiclassical approximation is obtained and justified in the case of impedance boundary conditions with a non zero absorbing part. It is shown that the total momentum transmitted to the obstacle vanishes when the frequency $k$ goes to infinity, and that the total cross section oscillates at high frequencies. When the impedance $\lambda_{0}$ is real (i. e., there is no absorption), it is shown that there exists a sequence of frequencies $k_{n}$ such that the averages in the impedance of the total cross section over shrinking intervals around $\lambda_{0}$ go to zero as $k_{n} \rightarrow \infty$.
\end{abstract}

Key words: invisible body, scattering by obstacles, total cross section, high frequency asymptotics, eikonal, Kirchhoff approximation.

AMS subject classification: 34L25, 58J50, 78A45, 34E20

*Department of Mathematics, Aveiro University, Aveiro 3810, Portugal. This work was supported by Centre for Research on Optimization and Control (CEOC) from the "Fundação para a Ciência e a Tecnologia" (FCT), cofinanced by the European Community Fund FEDER/POCTI, and by the FCT research project PTDC/MAT/113470/2009 (lakshtanov@rambler.ru).

†School of Mathematics, University of Leeds, Leeds, LS2 9JT,UK (bds@maths.leeds.ac.uk)

$\ddagger$ Department of Mathematics and Statistics, University of North Carolina, Charlotte, NC 28223, USA. The work was partially supported by the NSF grant DMS-1008132 (brvainbe@uncc.edu). 


\section{Introduction}

An interesting geometrical object was studied in a recent publication by Aleksenko and Plakhov [1]. This object $\mathcal{O}$ has the following property. Geometrical optical rays, coming from a particular direction and reflected twice from the boundary of $\mathcal{O}$ by the law of geometrical optics, continue to propagate parallel to each other in the same way as if the obstacle was absent. The object has zero classical total scattering cross section and appears invisible to an observer on the basis of the theory of geometrical optical rays. Note that a phase shift may influence the "invisibility" of the obstacle. One should also note that optical ray considerations provide an approximation to the expected properties of the corresponding optical problem, when the obstacle is smooth and convex. These conditions do not hold for the object under consideration. A rigorous treatment of the problem has to be based on an investigation of the solutions of the wave equation.

This paper concerns the study of the associated scattering problem for the reduced wave (i. e., Helmholtz) equation. High frequency asymptotics are obtained for the scattering of plane wave by the Aleksenko-Plakhov obstacle $\mathcal{O}$. The rigorous justification will be obtained in the case of impedance boundary conditions with a non-zero absorption. It will also be obtained in a weaker sense for boundary conditions without absorption. It is important to note that the delay time is the same for all the rays meeting the obstacle, i.e., the phase shift $\Delta$ is a constant for all rays meeting $\mathcal{O}$. In the absence of absorption, this implies that the obstacle $\mathcal{O}$ is almost invisible at a sequence of high frequencies $k=k_{n}=\frac{k^{0}+2 \pi n}{\Delta}, n \rightarrow \infty$, where $k^{0}$ is determined by the boundary condition, and the invisibility effect disappears for other frequencies.

Note that the scattering theory prohibits the existence of absolutely invisible bodies, since a nontrivial outgoing solution of the Helmholtz equation can not have zero scattering amplitude. Our results show that the scattering data for one incident direction and a sequence of frequencies can be as small as we please for an obstacle of an arbitrary size. Another by-product of our results concerns the relation between the total scattering cross section and the geometrical cross section. For the obstacle under consideration, the total scattering cross section approaches to four times the geometrical cross section for some increasing sequence of frequencies (when the incident and the reflected waves are in-phase), while for convex obstacles the corresponding ratio approaches to two as the frequency increases.

The simplicity of the model under consideration allows one to consider it as a candidate in competition with other models on cloaking (eg. [12]). Its obvious disadvantage, namely the incident direction is fixed, may be non- 
essential in some applications. On the other hand, the corresponding cloaking object is easy to make, in contrast to other cloaking materials which present formidable technological difficulties. Indeed currently proposed cloaking materials which lead to zero total scattering cross section for all incident directions need to have an infinite mass [12] or to be highly non-isotropic [3, 14]. The latter leads to huge difficulties in a practical realization of such a nonisotropic coating which are not attainable at present.
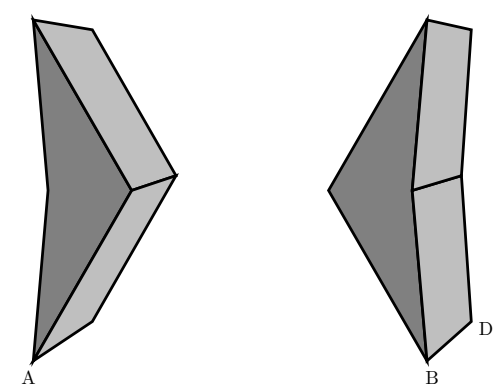

Figure 1: Aleksenko-Plakhov object $\mathcal{O}$. Translation distance $|B D|=1$.

The Aleksenko-Plakhov obstacle $\mathcal{O} \subset \mathbb{R}^{3}$ with Lipschitz boundary $\partial \mathcal{O}$ considered in this paper is presented in Fig. 1. It is formed by an orthogonal translation of the 2D object shown in Fig. 2. The prolongation of this translation is 1. In Fig. $2 A^{\prime} C^{\prime} B^{\prime}$ and $A C B$ are equilateral triangles. where $C, C^{\prime}$ are the mid points of segments $A^{\prime} B^{\prime}$ and $A B$, respectively. The lateral sides $A^{\prime} A$ and $B^{\prime} B$ are slightly displaced in order to avoid a positive measure of the obstacle's surface on the tangent rays propagating along the $z$-axis. We suppose that $A^{\prime} B^{\prime}=1$ that means that geometrical cross section of the body i. e.,the area of the projection of the body on the $(x, y)$-plane, equals to $1 / 2$.

The geometry of this obstacle is chosen in such a way that the geometrical optical rays propagating from the $z$-direction, after a double reflection from the boundary of the obstacle, continue to move parallel to each other with a constant shift $\Delta$ of the phase. This creates the effect of invisibility for an observer who relies only on geometrical optical rays coming from above (we discuss this in more detail in the next section). In fact, the geometry of triangles $A^{\prime} C^{\prime} B^{\prime}$ and $A C B$ can be chosen more generally, and other obstacles also can be constructed which also create the same effect of classical invisibility [1].

The goal of this paper is to study this invisibility effect using a rigorous analysis based on the investigation of the corresponding wave problem. It is well known that the justification of the geometrical optics approximation is a difficult task. This was done earlier in two cases: for smooth strictly convex obstacles [7, 10, 11, 6, 2] and for inhomogeneous media in the whole 
space [15]. For example in the first case, a complicated approximation of the field near the tangent rays is needed, and delicate local energy estimates are used. The latter are known for strictly convex or star-shaped obstacles. Some conditional results for non-convex obstacle can be found in [13] where the scattering amplitude is studied along the non-caustic directions.

Let $k>0$, the scattered field $u$ is a solution of the Helmholtz equation satisfying the Sommerfeld radiation condition:

$$
\begin{gathered}
\Delta u(r)+k^{2} u(r)=0, \quad r \in \Omega=\mathbb{R}^{3} \backslash \mathcal{O}, \\
\int_{|r|=R}\left|\frac{\partial u(r)}{\partial|r|}-i k u(r)\right|^{2} d S=o(1), \quad R \rightarrow \infty .
\end{gathered}
$$

We assume an impedance boundary condition holds on $\partial \mathcal{O}$, i.e.

$$
\left(\frac{\partial}{\partial n}+k \lambda\right)\left(u+e^{i k\left(r \cdot p_{0}\right)}\right)=0, \quad r=(x, y, z) \in \partial \mathcal{O}, \quad p_{0}=(0,0,1),
$$

where $\Im \lambda \geq 0$ is a constant and $n$ denotes the outer unit normal for $\mathcal{O}$. There exists a unique solution in $H_{l o c}^{1}(\Omega)$ which satisfies all these conditions (eg [9]). Every solution $u(r)$ of (11)-(3) has the following behavior at infinity

$$
u(r)=\frac{e^{i k|r|}}{|r|} u_{\infty}(\theta)+o\left(\frac{1}{|r|}\right), \quad r \rightarrow \infty, \quad \theta=r /|r| \in S^{2},
$$

where the function $u_{\infty}(\theta)=u_{\infty}(\theta, k)$ is called the scattering amplitude and the quantity

$$
\sigma(k)=\left\|u_{\infty}\right\|_{L_{2}\left(S^{2}\right)}^{2}=\int_{S^{2}}\left|u_{\infty}(\theta)\right|^{2} d \mu(\theta)
$$

is called the total cross section. Here $d \mu$ is the surface element of the unit sphere.

The following observable is called the transport cross section.

$$
\left.\sigma_{T}(k)=\int_{S^{2}}\left(1-\theta \cdot p_{0}\right)\right)\left|u_{\infty}(\theta)\right|^{2} d \mu(\theta)
$$

and equals the total momentum transmitted to the obstacle per unit time in large volume normalization.

The following are the main results obtained in this paper. They are stronger if $\Im \lambda>0$ (i. e., there is absorption of the energy at the boundary), and hold on average if $\Im \lambda=0$. 
Theorem 1. Let $\Im \lambda>0$. Then

1) the transport cross section vanishes as $k$ goes to infinity:

$$
\lim _{k \rightarrow \infty} \sigma_{T}=0,
$$

2) the total cross section has the following asymptotic behavior for large $k$ :

$$
\sigma(k)=\frac{1}{2}\left|A^{2} e^{i k \Delta}-1\right|^{2}+o(1), \quad k \rightarrow \infty, \quad A=\frac{i-2 \lambda}{i+2 \lambda},
$$

where $\Delta$ is defined in Fig. 2 .

3) $\left|u_{\infty}(\theta)\right|^{2} \sim \sigma(k) \delta\left(p_{0}\right), \quad k \rightarrow \infty$ in the sense of distributions, i.e., for any $\varphi \in C\left(S^{2}\right)$, we have

$$
\int_{S^{2}} \varphi(\theta)\left|u_{\infty}(\theta)\right|^{2} d S_{\theta}=\frac{1}{2}\left|-1+A^{2} e^{i k \Delta}\right|^{2} \varphi\left(p_{0}\right)+o(1), \quad k \rightarrow \infty .
$$

Remark. If $\lambda$ is real, then $|A|=1$. Thus if the second statement holds for real $\lambda$ then

$$
\sigma(k)=\frac{1}{2}\left|-1+e^{i(k \Delta+2 \arg (A))}\right|^{2}+o(1), \quad k \rightarrow \infty .
$$

and

$$
\lim _{n \rightarrow \infty} \sigma\left(k_{n}\right)=0 \text { for } k_{n}=\frac{-2 \arg (A)}{\Delta}+\frac{2 \pi}{\Delta} n, \quad n \in \mathbb{Z}, \quad \Im \lambda=0 .
$$

The almost invisibility of $\mathcal{O}$ manifests itself by the fact that the total cross section $\sigma(k)$ can be made as small as we please by choosing a complex impedance $\lambda$ close enough to an arbitrary real $\lambda=\lambda_{0}$ and then choosing $k=k_{n}$ large enough.

It also follows from (6) with real $\lambda$ that $\sigma(k) \rightarrow 2$ when $k=\frac{-2 \arg (A)}{\Delta}+$ $\frac{(2 n+1) \pi}{\Delta} \rightarrow \infty$, i.e., $\sigma(k)$ approaches four times the geometrical cross section. The latter is the area of the shadow of the obstacle if the reflected rays are not taken into account, and this area equals $1 / 2$. For convex obstacles, the ratio of the total to the geometrical cross section approaches two as $k \rightarrow \infty$.

In some cases we will denote the total cross section (5) by $\sigma_{\lambda}(k)$ in order to stress its dependence on the value of the parameter $\lambda$ in the boundary condition (3).

Theorem 2. Let $\lambda_{0}$ be real and

$$
k_{n}=\frac{-2 \arg \left(A\left(\lambda_{0}\right)\right)}{\Delta}+\frac{2 \pi}{\Delta} n, \quad n \in \mathbb{Z}
$$


Then there exists a sequence of positive numbers $\varepsilon_{n} \rightarrow 0, n \rightarrow \infty$, such that

$$
\frac{1}{\varepsilon_{n}} \int_{\lambda_{0}-\varepsilon_{n}}^{\lambda_{0}+\varepsilon_{n}} \sigma_{\lambda}\left(k_{n}\right) d \lambda \rightarrow 0, \quad n \rightarrow \infty .
$$

Consequence. Under conditions of Theorem 2, there exists a sequence of real numbers $\lambda_{n} \rightarrow \lambda_{0}$ such that $\lim _{n \rightarrow \infty} \sigma_{\lambda_{n}}\left(k_{n}\right)=0$.

The paper is organized as follows. The eikonal geometrical optics approximation is constructed in the next section. It provides a basis to understand the validity of Theorems 1, 2, It is also needed in order to construct the Kirchhoff approximation $u^{0}$ which is used to prove these results. Section 3 outlines the proof of Theorem 1. There we state the asymptotic properties of the Kirchhoff approximation in a bounded region and discuss the estimates needed to justify the approximation. In order to obtain the properties of $u^{0}$ we study first Kirchhoff approximation for the problem of scattering by a single polygon (one face of the obstacle $\mathcal{O}$ ). This is done in section 4 with the main technical parts of the proof moved into Appendix 1. The asymptotic behavior in a bounded region of the Kirchhoff approximation for the obstacle $\mathcal{O}$ is obtained in section 5 . Sections 6 and 7 provide the asymptotic behavior of the total and transport cross sections, respectively, when $\Im \lambda>0$. The case $\Im \lambda=0$ is studied in section 8 .

\section{Eikonal approximation}

We begin by recalling that the construction of the eikonal approximation to the solution of the scattering problem uses a Lagrangian manifold $\Lambda$ formed by trajectories $(r, p)=(r(t), p(t))$ in the phase space $\mathbb{R}^{3} \times \mathbb{R}^{3}$ which correspond to the geometrical optics rays $r=r(t)$. The components of the vector $(r, p)$ are the position $r=r(t)$ of a point along the ray at time $t$ and the momentum $p=p(t)$ (where $p$ is the unit vector along the ray). The trajectories start at time $t=0$ at points $\left(x_{0}, y_{0},-a\right)$ of the plane $z=-a$ with momentum $p_{0}=(0,0,1)$. It is supposed that the obstacle $\mathcal{O}$ is situated in the half space $z>-a$. Location and momentum $(r, p)$ of every ray, except those which meet an edge of $\mathcal{O}$, are determined uniquely for all $t \in \mathbb{R}$. Thus, $\left(x_{0}, y_{0}, t\right) \in \mathbb{R}^{3}$ are global coordinates on $\Lambda$. Let $P_{r}: \Lambda \rightarrow \mathbb{R}^{3}$ be the projection of $\Lambda$ to the coordinate space $\mathbb{R}^{3}$ and let $\mathcal{B}=P_{r} \partial \Lambda \subset \mathbb{R}^{3}$.

In the case of the Neumann boundary condition, the eikonal geometrical optics approximation has the form:

$$
\Psi^{e i k}(r)=\sum_{\left(x_{0}, y_{0}, t\right): r\left(x_{0}, y_{0}, t\right)=r}\left|\frac{D(r)}{D\left(x_{0}, y_{0}, t\right)}\right|^{-1 / 2} e^{i k S\left(x_{0}, y_{0}, t\right)}, \quad r \in \mathbb{R}^{3} \backslash \mathcal{B} .
$$


Here the action $S$ is just the length of the trajectory, i.e. $S(x, y, t)=t$ (since the exterior of the obstacle is homogeneous), the Jacobian $\left|D(r) / D\left(x_{0}, y_{0}, t\right)\right|$ is the density of geometrical optics rays in ray tubes, and the summation is taken over all the points of $\Lambda$ having $r$ as projection to $\mathbb{R}^{3}$.

Function $\Psi^{e i k}$ satisfies (3) outside $\mathcal{B}$ and is discontinuous on $\mathcal{B}$. Nevertheless, we can use it to predict quasi-classic effects. Note that reflection from the plane faces of $\mathcal{O}$ does not change the density of the rays and so

$$
\Psi^{e i k}(r)=\sum_{\left(x_{0}, y_{0}, t\right): r\left(x_{0}, y_{0}, t\right)=r} e^{i k t}, \quad r \in \mathbb{R}^{3} \backslash \mathcal{B} .
$$

In Figure 2 (where $A=1$ in the case of Neumann or $A=-1$ in the case of Dirichlet boundary conditions) we see that the obstacle does not have a shadow: for example, the shadow zone of the right part of the obstacle is covered by the rays reflected from $A^{\prime} A^{\prime \prime}$ and $B^{\prime \prime} B$.

The following observation on the geometrical structure is important: each trajectory started on the line $A^{\prime} B^{\prime}$ between points $A^{\prime}$ and $G$ or between point $H$ and $B^{\prime}$ and ended on the line $A B$ have the same length. It means that all the rays below the line $A B$ have momentum $p_{0}=(0,0,1)$, the action for the rays coming through $G H$ is $z$, and the action for the rays which have collisions with the obstacle is $\Delta+z$, where $\Delta=\left|G A^{\prime \prime}\right|+\left|A^{\prime \prime} B\right|-\left|A^{\prime} A\right|$. So the rays below the line $A B$ differ from the sets of rays in the absence of the obstacle only by the phase shift $\Delta$ on the rays coming through $A^{\prime} H \cup G H^{\prime}$. Hence, if $k \Delta$ is a multiple of $2 \pi$, the eikonal approximation outside of some neighborhood of the obstacdikr coin Gides withrthe incidentekvave. The scattering

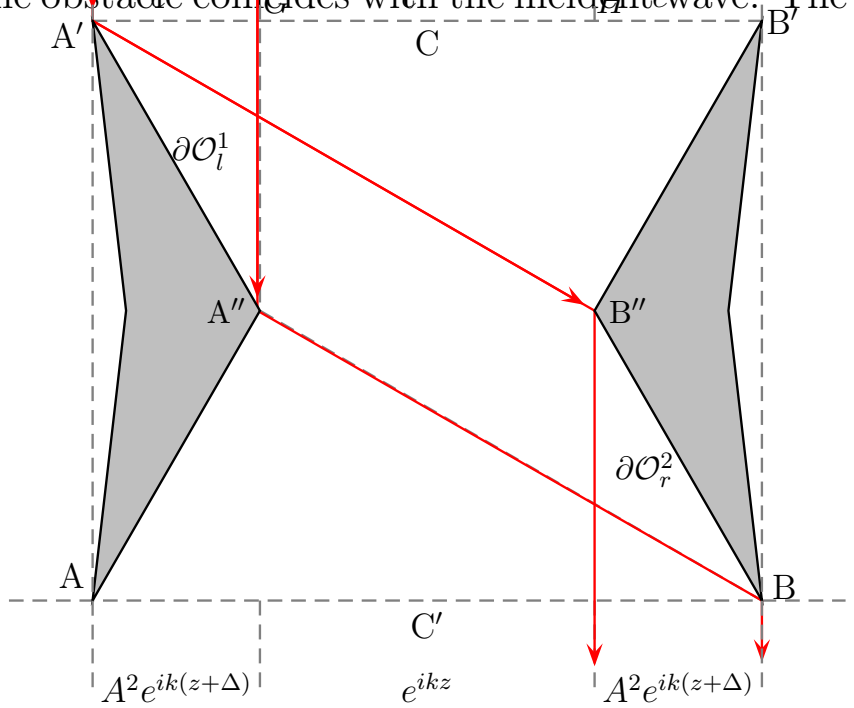

Figure 2: Plane section of $\mathcal{O}$. Base side $\left|A^{\prime} B^{\prime}\right|=1$. Eikonal approximation $\Psi^{e i k}$ is given above $A^{\prime} B^{\prime}$ and below $A B ; \Delta=\left|G A^{\prime \prime}\right|+\left|A^{\prime \prime} B\right|-\left|A^{\prime} A\right|$. 
amplitude is not defined for the eikonal approximation, however the transport cross section for the eikonal approximation is defined by the following limit: $\lim _{R \rightarrow \infty} \int_{|r|=R}\left(1-\theta \cdot p_{0}\right)\left|\Psi^{e i k}(r)-e^{i k r \cdot p_{0}}\right|^{2} d S$.

Since all rays have forward direction after collisions with the obstacle, the transport cross section for the eikonal approximation is zero. The arguments above concern the case of the Neumann boundary condition $(\lambda=0)$. When the impedance $\lambda$ is arbitrary, the terms in the eikonal approximation (9) after each collision are multiplied by the factor

$$
\frac{i n_{\alpha}+\lambda}{i n_{\alpha}-\lambda}
$$

where $n_{\alpha}=n \cdot \alpha$ is the cosine of the angle between the normal to the surface at the point of the incidence and the direction $\alpha$ of the ray before the incidence. In the case of our obstacle $\mathcal{O}$ all incidences occur with the same $n_{\alpha}=-1 / 2$.

Define $A(\lambda):=\frac{i-2 \lambda}{i+2 \lambda}$, then the eikonal approximation in the case of the impedance boundary condition can be written as

$$
\Psi^{e i k}(r)=\sum_{\left(x_{0}, y_{0}, t\right): r\left(x_{0}, y_{0}, t\right)=r}[A(\lambda)]^{n\left(x_{0}, y_{0}, t\right)} e^{i k t}, \quad r \in \mathbb{R}^{3} \backslash \mathcal{B} .
$$

where $n\left(x_{0}, y_{0}, t\right)$ denotes the number of collisions of the ray with initial coordinates $\left(x_{0}, y_{0}\right)$ with $\partial \mathcal{O}$ before time $t$. Thus the field behind the triangles (after two collisions) is $A^{2} e^{i k(z+\Delta)}$ (see Fig. 2).

Note that the factor (11) is chosen by the requirement for $\Psi^{e i k}(r)$ to satisfy the impedance boundary condition, i.e.

$$
\left(\frac{\partial}{\partial n}+k \lambda\right) \Psi^{e i k}(r)=0, \quad r \in \partial \mathcal{O} .
$$

\section{Outline of the proof of Theorem 1}

The main technical difficulties of the present paper concern the construction of an appropriate asymptotic approximation for the scattered field. We will not work directly with $\Psi^{e i k}$ which has jumps and does not satisfy equation (11). Instead we use the Kirchhoff approximation $u^{0}$ to the solution $u$ in order to prove Theorem 2. This necessitates the need to justify the validity of the Kirchhoff approximation and to study its asymptotic behavior.

Recall that the Green formula allows one to represent field $u$ in terms of a surface integral involving $\left.u\right|_{\partial \mathcal{O}},\left.\frac{\partial u}{\partial n}\right|_{\partial \mathcal{O}}$. If $\partial \mathcal{O}$ were smooth, the Kirchhoff approximation would be given by the same Green formula with $u$ and its 
derivative in the integrands are replaced by $\psi^{e i k}(r):=\Psi^{e i k}(r)-e^{i k r \cdot p_{0}}$ and its derivative, respectively, i.e.,

$$
u^{0} \approx \frac{1}{4 \pi} \int_{\partial \mathcal{O}}\left[-\frac{\partial}{\partial n_{q}}\left(\psi^{e i k}(q)\right) \frac{e^{i k|r-q|}}{|r-q|}+\psi^{e i k}(q) \frac{\partial}{\partial n_{q}} \frac{e^{i k|r-q|}}{|r-q|}\right] d S_{q} .
$$

The integral above with $e^{i k r \cdot p_{0}}$ instead of $\psi^{e i k}$ is equal to zero. Thus

$$
u^{0} \approx \frac{1}{4 \pi} \int_{\partial \mathcal{O}}\left[-\frac{\partial}{\partial n_{q}}\left(\Psi^{e i k}(q)\right) \frac{e^{i k|r-q|}}{|r-q|}+\Psi^{e i k}(q) \frac{\partial}{\partial n_{q}} \frac{e^{i k|r-q|}}{|r-q|}\right] d S_{q} .
$$

The exact definition of $u^{0}$, in our case where $\partial \mathcal{O}$ is non-smooth, differs only by the introduction of a cut-off function $\eta=\eta(k, q)$ in the integrand above which makes the integrand smoother and provides important uniform estimates of $u^{0}$. Thus

$$
u^{0}=\frac{1}{4 \pi} \int_{\partial \mathcal{O}} \eta\left[-\frac{\partial}{\partial n_{q}}\left(\Psi^{e i k}(q)\right) \frac{e^{i k|r-q|}}{|r-q|}+\Psi^{e i k}(q) \frac{\partial}{\partial n_{q}} \frac{e^{i k|r-q|}}{|r-q|}\right] d S_{q} .
$$

Here $\eta$ is a $C^{\infty}$-function defined on the faces of the polyhedron $\partial \mathcal{O}$ which vanishes in a $k^{-\delta}$-neighborhood of the edges of $\partial \mathcal{O}$, equals one outside of a $2 k^{-\delta}$-neighborhood of the edges, and is such that $\left|D^{m} \eta\right|<C_{m} k^{\delta m}, k>1$, for each partial derivative $D^{m}$ of order $m$ on faces of $\partial \mathcal{O}$. One can choose $\delta$ arbitrarily in the interval $0<\delta<1 / 2$. This choice of $\delta$ allows one to apply the stationary phase method when an amplitude contains the factor $\eta$. However, some of the arguments at the end of section 7 become simpler when $\delta$ is small enough. Indeed it is sufficient to fix $\delta=1 / 4$ and so

$$
\left|D^{m} \eta\right|<C_{m} k^{m / 4}, \quad k>1 .
$$

The function $u^{0}$ satisfies (1), (2). Furthermore, it will be proved in section 5 that the following statement holds

\section{Lemma 1.}

$$
\left\|\left.\left(\frac{\partial}{\partial n}+k \lambda\right)\left(u^{0}(r)-u(r)\right)\right|_{\partial \mathcal{O}}\right\|_{L_{2}(\partial \mathcal{O})}=o(k), \quad k \rightarrow \infty .
$$

This Lemma together with an a priori estimate for the solutions of the problem (11)-(3) with $\Im \lambda>0$ obtained in [4], allows one to justify the approximations $u \sim u^{0}, u_{\infty} \sim u_{\infty}^{0}, k \rightarrow \infty$. All the theorems in [4] were formulated for quite smooth obstacles. However, the proof of the Theorem 2 of that paper is based only on an application of the Green formula, which is valid for piecewise smooth obstacles also. Due to the importance (and simplicity) of this theorem for our purposes, we prove a slightly improved version of this theorem here. 
Theorem 3. Let the function $v$ satisfy (11), (2) and the boundary condition

$$
\left(\frac{\partial}{\partial n}+k \lambda\right) v=f, \quad r=(x, y, z) \in \partial \mathcal{O}, \quad \Im \lambda>0 .
$$

Then

$\left\|v_{\infty}\right\|_{L_{2}\left(S^{2}\right)} \leq \frac{1}{2 k \sqrt{\Im \lambda}}\|f\|_{L_{2}(\partial \mathcal{O})},\|v\|_{L_{2}(\partial \mathcal{O})}+\left\|\frac{1}{k \lambda} \frac{\partial v}{\partial n}\right\|_{L_{2}(\partial \mathcal{O})} \leq \frac{1}{k \Im \lambda}\|f\|_{L_{2}(\partial \mathcal{O})}$.

Proof. Taking the imaginary parts of both sides of the Green formula

$$
\int_{\Omega}\left(\Delta v+k^{2} v\right) \bar{v} d x=\int_{\partial \Omega} v_{n} \bar{v} d S-i k \int_{S^{2}}\left|v_{\infty}\right|^{2} d S-\int_{\Omega}\left(|\nabla v|^{2}-k^{2}|v|^{2}\right) d x
$$

we obtain $\Im \int_{\partial \Omega} v_{n} \bar{v} d S-k \int_{S^{2}}\left|v_{\infty}\right|^{2} d S=0$. Thus, for any $c>0$,

$k \Im \lambda \int_{\partial \Omega}|v|^{2} d S+k \int_{S^{2}}\left|v_{\infty}\right|^{2} d S \leq \int_{\partial \Omega}|f v| d S \leq c k \Im \lambda \int_{\partial \Omega}|v|^{2} d S+\frac{1}{4 c k \Im \lambda} \int_{\partial \Omega}|f|^{2} d S$.

Choosing $c=1$, or $c=1 / 2$ we arrive to the first inequality (17) or to the second inequality for $v$, respectively. The second inequality for $v_{n}$ follows from the boundary condition. The proof is complete.

Lemma 1 and Theorem 3 imply

$$
\left\|u_{\infty}^{0}-u_{\infty}\right\|_{L_{2}\left(S^{2}\right)}=o(1), \quad k \rightarrow \infty, \quad \Im \lambda>0
$$

Evidently, for any $\varphi \in C\left(S^{2}\right)$ we have

$$
\int_{S^{2}} \varphi(\theta)\left|u_{\infty}(\theta)\right|^{2} d S_{\theta}=\int_{S^{2}} \varphi(\theta)\left|u_{\infty}^{0}(\theta)\right|^{2} d S_{\theta}+o(1), \quad k \rightarrow \infty, \quad \Im \lambda>0 .
$$

Hence, to prove Theorem 1 we need only prove Lemma 1 and to analyze the far field behavior of $u^{0}$.

Lemma 1 and the far field behavior of $u^{0}$ will be derived from the following lemma on the near field behavior of the Kirchhoff approximation. To state the lemma we need to introduce the set $\widehat{\mathcal{B}}$ which is formed by extended to infinity lateral boundaries of shadow and reflected regions. One can define $\widehat{\mathcal{B}}$ as follows. Referring to Fig. 2, let $\partial \mathcal{O}^{1}$ be the upper part of $\partial \mathcal{O}$ which is struck by the incident wave, and let $\partial \mathcal{O}^{2}$ be the lower part of $\partial \mathcal{O}$ located strictly below $\partial \mathcal{O}^{1}$ (it is struck by the reflected wave). Their left and right halves (rectangles) will be denoted by $\partial \mathcal{O}_{s}^{i}, i=1,2 ; s \in\{l, r\}$. Every time a geometrical optics ray strikes (directly or after a reflection) an edge of one of the four faces $\partial \mathcal{O}_{s}^{i}, i=1,2 ; s \in\{l, r\}$ of the obstacle $\mathcal{O}$, we extend the 
ray to infinity in both directions. That is in the direction of the incident ray and in the direction of rays reflected from the face. These extensions form $\widehat{\mathcal{B}}$. Consider, for example the incident ray $\left(G A^{\prime \prime}\right)$ on Fig. 2 . Then $\widehat{\mathcal{B}}$ contains half-infinite ray $\left(A^{\prime \prime} B\right)$ starting at $A^{\prime \prime}$ and half-infinite ray starting at $B$ and propagating downwards.

Lemma 2. The following asymptotics hold uniformly on any compact of $\partial \mathcal{O}$ which does not contain points of edges and on any compact set in $\mathbb{R}^{3} \backslash \widehat{\mathcal{B}}$ :

$$
u^{0}(r)=\psi^{e i k}(r)+O(1 / \sqrt{k}), \quad\left|\nabla u^{0}(r)-\nabla \psi^{e i k}(r)\right|=O(\sqrt{k}), \quad k \rightarrow \infty .
$$

For any $R>0$ there exist constants $C_{i}=C_{i}(R), i=1,2$ such that

$$
\left|u^{0}(r)-\psi^{e i k}(r)\right|<C_{1}, \quad\left|\nabla u^{0}(r)-\nabla \psi^{e i k}(r)\right|<k C_{2}
$$

when $|r|<R, \quad k \rightarrow \infty$.

Remark. Note that $\Psi^{e i k}$ has jumps on $\mathcal{B}$. In all estimates involving $\Psi^{e i k}$ on $\partial \mathcal{O}$ or $\mathcal{B}$ we assume that one-sided limits of the corresponding functions are considered.

Lemmas 2 and 1 will be proved in section 5. After that Theorem 1 follows from the following two well known formulas:

$$
\begin{gathered}
u_{\infty}^{0}(\theta)=\frac{-1}{4 \pi} \int_{Q}\left[\frac{\partial u^{0}}{\partial n}(r)+i k\left(\frac{r}{|r|} \cdot \theta\right) u^{0}\right] e^{-i k(\theta \cdot r)} d S, \quad \theta \in S^{2}, \\
\left\|u_{\infty}^{0}\right\|_{L_{2}\left(S^{2}\right)}=\frac{1}{k} \Im \int_{Q} \frac{\partial u^{0}}{\partial n} \overline{u^{0}} d S,
\end{gathered}
$$

where $Q$ is a closed surface such that the bounded part of the space with the boundary $Q$ contains $\mathcal{O}$. Note, one can take $Q=\partial \mathcal{O}$.

\section{Scattering by a polygon $M \subset \mathbb{R}^{3}$}

This section is devoted to a study of a simplified version of the problem under consideration. It concerns the scattering by a single polygon which represents a typical face of the polyhedron $\mathcal{O}$. The obtained results will be used later when the polyhedron $\mathcal{O}$ is considered.

Let $P \subset \mathbb{R}^{3}$ be a plane in $\mathbb{R}^{3}$, and let $\alpha \in S^{2}$ be a unit vector which is transversal to $P$. We choose a unit normal vector $n \in S^{2}$ to $P$ in such a way that $n_{\alpha}=\cos \theta=(n, \alpha)<0$.

Let $M$ be a convex domain in $P$ bounded by a polygon $\partial M$. By the shadow zone $S(\alpha)=S(\alpha, M) \subset \mathbb{R}^{3}$ we mean the domain which is inaccessible 
for the direct ray propagating along the vector $\alpha$ assuming that $P \backslash M$ is transparent and that $M$ reflects the ray, i.e., $S(\alpha)=\left\{r=r_{0}+t \alpha \in \mathbb{R}^{3}: r_{0} \in\right.$ $M, t>0\}$. We define the reflected zone to be the area $R(\alpha)=R(\alpha, M) \subset \mathbb{R}^{3}$ covered by the reflected rays, i.e., $R(\alpha)=\left\{r=r_{0}+t \alpha^{*} \in \mathbb{R}^{3}: r_{0} \in M, t>\right.$ $0\}$, where $\alpha^{*}=\alpha^{*}(M)=\alpha-2(\alpha \cdot n) n \in S^{2}$ is the reflection direction. In future $\alpha$ will be always equal to $p_{0}$ (see Fig. 3) or $p_{0}^{*}$ (see Fig. 4).

Denote by $D(r)=D_{\alpha, M}(r)$ the single layer potential with density $\eta e^{i k(\alpha \cdot r)}$ :

$$
D(r)=\int_{M} \eta e^{i k(\alpha \cdot q)} \frac{e^{i k|r-q|}}{|r-q|} d S(q), \quad r \in \mathbb{R}^{3},
$$

where $\eta$ is the function introduced in (14). For any $r \in M$, define $r_{\varepsilon}=r+n \varepsilon$, $\varepsilon>0$ and let

$$
\frac{\partial}{\partial n} D(r)=\lim _{\varepsilon \rightarrow 0} \frac{\partial}{\partial n} D\left(r_{\varepsilon}\right)
$$

Denote by $N(r)=N_{\alpha M}(r)$ the double layer potential with density $\eta e^{i k(\alpha \cdot r)}$ :

$$
N(r)=\int_{M} \eta e^{i k(\alpha \cdot q)} \frac{\partial}{\partial n_{q}} \frac{e^{i k|r-q|}}{|r-q|} d S(q), \quad q \notin M,
$$

and define its value on $M$ similar to (25), i.e.

$$
N(r)=\lim _{\varepsilon \rightarrow 0} N\left(r_{\varepsilon}\right), \quad \frac{\partial}{\partial n} N(r)=\lim _{\varepsilon \rightarrow 0} \frac{\partial}{\partial n} N\left(r_{\varepsilon}\right), \quad r \in M .
$$

Finally define $A=A(\alpha, M)=\frac{i(n \cdot \alpha)+\lambda}{i(n \cdot \alpha)-\lambda}$ (see (11) $)$.

Consider scattering of $e^{i k r \cdot \alpha}$ by the obstacle consisting of the surface $M$ only, and define the eikonal approximation $\Psi_{\alpha, M}^{e i k}$ as in (12). Thus (see Fig. 31)

$$
\psi_{\alpha, M}^{e i k}(r):=\Psi_{\alpha, M}^{e i k}(r)-e^{i k r \cdot \alpha}=\left\{\begin{array}{cc}
-e^{i k r \cdot \alpha}, & r \in S(\alpha, M) \\
A e^{i k\left[\left(\alpha^{*} \cdot r\right)+t_{0}\right]}, & r \in R(\alpha, M) \\
0, & \mathbb{R}^{3} \backslash\{R(\alpha) \cup S(\alpha)\}
\end{array}\right.
$$

where the constant $t_{0}=t_{0}(\alpha, M)$ is defined by the relation

$$
e^{i k\left[\left(\alpha^{*} \cdot r\right)+t_{0}\right]}=e^{i k(\alpha \cdot r)}, \quad r \in M .
$$

Since $\alpha^{*}=\alpha-2(\alpha \cdot n) n,(28)$ implies that

$$
t_{0}=2(\alpha \cdot n)(n \cdot r), \quad r \in M
$$

Note that $n \cdot\left(r_{1}-r_{2}\right)=0$ when $r_{1}, r_{2} \in M$. Thus, $t_{0}$ is a constant. 
The function $\Psi_{\alpha, M}^{e i k}(r)$ has one-sided limiting values on both sides of $M$, the value of the function and of its normal derivative is zero on the shadow side of $M$. On the illuminated side $M_{+}$of $M$ :

$$
\Psi_{\alpha, M}^{e i k}(r)=(A+1) e^{i k r \cdot \alpha}, \quad \frac{\partial}{\partial n} \Psi_{\alpha, M}^{e i k}(r)=i k(-A+1) n_{\alpha} e^{i k r \cdot \alpha}, \quad r \in M_{+} .
$$

Thus the Kirchhoff approximation (14) in the case of $M=\mathcal{O}$ takes the form

$$
\Phi=\Phi_{\alpha, M}=\frac{i k}{4 \pi}(A-1) n_{\alpha} D_{\alpha, M}+\frac{A+1}{4 \pi} N_{\alpha, M} .
$$

The following lemma plays a crucial role in the proof of the main result, theorem 1. Informally it states that the Kirchhoff approximation in the case of $\mathcal{O}=M$ is close to $\psi_{\alpha, M}^{e i k}:=\Psi_{\alpha, M}^{e i k}-e^{i k r \cdot \alpha}$ when $k \gg 1$, and it justifies Fig. 3 ,

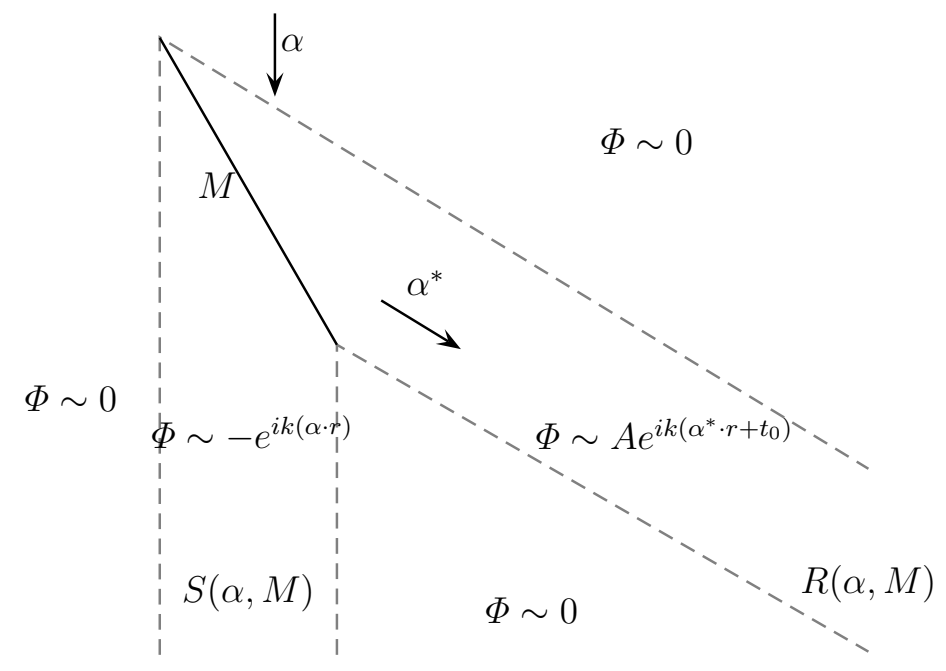

Figure 3: The figure presents the main term of the asymptotics of the Kirchhoff approximation $\Phi=\Phi_{\alpha, M}$ as $k \rightarrow \infty$. Here $R(\alpha, M)$ is the reflected zone, $S(\alpha, M)$ is the shadow zone, and $\Phi$ vanishes outside of $\overline{R(\alpha, M) \bigcup S(\alpha, M)}$ when $k \rightarrow \infty$. The main term of asymptotics coincides with $\psi_{\alpha, M}^{e i k}$. A rigorous statement is given by Lemma 3 .

Lemma 3. The following asymptotics as $k \rightarrow \infty$ hold uniformly on any compact set in $M$ and on any compact set of $\mathbb{R}^{3} \backslash \overline{R(\alpha, M) \cup S(\alpha, M)}$

$$
\Phi_{\alpha, M}(r)=\psi_{\alpha, M}^{e i k}(r)+O(1 / \sqrt{k}), \quad\left|\nabla \Phi_{\alpha, M}(r)-\nabla \psi_{\alpha, M}^{e i k}(r)\right|=O(\sqrt{k}) .
$$

For any $R>0$ there exist constants $C_{i}=C_{i}(R), i=1,2$ such that

$$
\left|\Phi_{\alpha, M}(r)-\psi_{\alpha, M}^{e i k}(r)\right|<C_{1}, \quad\left|\nabla \Phi_{\alpha, M}(r)-\nabla \psi_{\alpha, M}^{e i k}(r)\right|<k C_{2},
$$

when $|r|<R, k \rightarrow \infty$. 
Remarks. 1) Note that $\Psi_{\alpha, M}^{e i k}$ has jump discontinuities on the boundaries of the reflected and shadow zones. In all estimates involving $\Psi^{e i k}$ on $M$ or on the lateral sides of the boundaries of the reflected and shadow zones, we assume that one-sided limits of the corresponding functions are considered.

2) Since $\psi_{\alpha, M}$ satisfies the impedance boundary condition (3), the estimates (32), (33) imply that

$$
\frac{\partial}{\partial n} \Phi_{\alpha, M}+k \lambda \Phi_{\alpha, M}=-k(i(n \cdot \alpha)+\lambda) e^{i k(\alpha \cdot r)}+O(\sqrt{k}), k \rightarrow \infty,
$$

where the estimate of the remainder is uniform on any compact subset of $M$, and is bounded by $C k$ on $M$.

The proof of Lemma 3 is rather technical and is given in Appendix 1.

\section{Asymptotics of the Kirchhoff approxima- tion in a bounded region}

Proof of Lemma 2. Recall, see Fig. 2, that $\partial \mathcal{O}_{l}^{1}, \partial \mathcal{O}_{r}^{1}$ are the upper parts of the $\partial \mathcal{O}$ which are struck by the incident wave, and that $\partial \mathcal{O}_{l}^{2}, \partial \mathcal{O}_{r}^{2}$ are the lower parts of the $\partial \mathcal{O}$ which are struck by the reflected wave.

Note that function $\Psi^{e i k}$ in (14) vanishes on the part of the boundary of the obstacle which is not accessible for the rays, i.e., $\partial \mathcal{O}$ in (14) can be replaced by the union of four polygons $\partial O_{j}^{i}, i=1,2, j=l, r$. We split them in two pairs where the polygons in each pair are connected by rays. The first pair consists of $M_{1}=\partial O_{l}^{1}$ and $M_{2}=\partial O_{r}^{2}$. Then $u^{0}$ can be written in the form

$$
u^{0}=\mathcal{L}+\mathcal{R},
$$

where

$\mathcal{L}=-\frac{1}{4 \pi} \int_{M_{1} \cup M_{2}} \eta \frac{\partial}{\partial n_{q}}\left(\Psi^{e i k}(q)\right) \frac{e^{i k|r-q|}}{|r-q|} d S_{q}+\frac{1}{4 \pi} \int_{M_{1} \cup M_{2}} \eta \Psi^{e i k}(q) \frac{\partial}{\partial n_{q}} \frac{e^{i k|r-q|}}{|r-q|} d S_{q}$,

and $\mathcal{R}$ is given by a similar expression with $\partial O_{l}^{1} \bigcup \partial O_{r}^{2}$ in place of $M_{1} \bigcup M_{2}$. We represent $\mathcal{L}$ as $\mathcal{L}=\Phi_{1, l}+\Phi_{2, r}$ where the first term in the sum involves integration only over $M_{1}$ and the second term involves integration only over $M_{2}$. Then $\Phi_{1, l}$ coincides with the Kirchhoff approximation $u^{0}$ for a single polygon which is given by (31) as studied in the previous section. In particular, Fig. 3 and Lemma 3 with $\alpha=p_{0}$ are valid for $\Phi_{1, l}$.

Function $\Psi^{e i k}$ in the region covered by the rays between $M_{1}$ and $M_{2}$ has the form of a plane wave propagating in the direction $p_{0}^{*}$, i.e., $\Phi_{2, r}$ is 


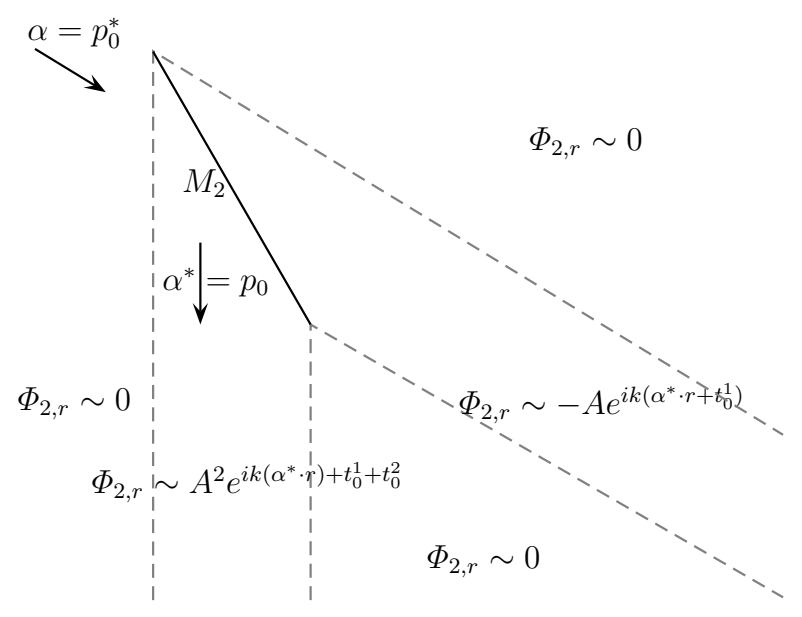

Figure 4: The main term of asymptotics of $\Phi_{2, r}$

also a Kirchhoff approximation for a single polygon. The only difference between $\Phi_{1, r}$ and $\Phi_{2, r}$ is that in the second case the direction $\alpha$ of the incident wave is different $\left(\alpha=p_{0}^{*}\right)$ and the incident wave contains a constant factor $A e^{i k t_{0}}$. Thus, the main term of asymptotics of $\Phi_{2, r}$ is given by Fig. 4. This figure coincides with Fig. 3 with $\alpha=p_{0}^{*}$ and extra factor $A e^{i k t_{0}}$ added. An analogue of Lemma 3 is valid for $\Phi_{2, r}$ stating that $\Phi_{2, r}$ differs from the main term indicated in Fig. 4 by $O(1 / \sqrt{k})$ on any compact outside of the lateral sides of reflected and shadow zones and by $O(1)$ on any compact set. The remainder is multiplied by $k$ after differentiation. Thus, the main term of $\mathcal{L}=\Phi_{1, l}+\Phi_{2, r}$ is indicated in Fig. 5. The shift of the phase $t_{0}^{1}+t_{0}^{2}$ in Fig. 5 was discussed in section 2 , where it was noted that this shift is equal to $\Delta$ (see Fig. 2). Since $\mathcal{R}$ has a similar asymptotic expansion and $u^{0}=\mathcal{L}+\mathcal{R}$, this proves the statements of Lemma 2 .

Proof of Lemma 1. From (3) and (11) it follows that

$$
\left(\frac{\partial}{\partial n}+k \lambda\right)\left(u-\psi^{e i k}(r)\right)=0, \quad r \in \partial \mathcal{O} .
$$

On the other hand, Lemma 2 shows that $u^{0}$ is close to $\psi^{e i k}(r)$ near $\partial \mathcal{O}$. This leads to the statement of Lemma 1. Indeed, let us fix an arbitrary $\varepsilon>0$ and let $\partial \mathcal{O}_{\varepsilon}$ be such a small neighborhood of the edges on $\partial \mathcal{O}$ that the area $\left|\partial \mathcal{O}_{\varepsilon}\right|$ of that neighborhood does not exceed $\frac{\varepsilon^{2}}{2\left(C_{1}|\lambda|+C_{2}\right)^{2}}$, where $C_{1}, C_{2}$ are constants defined in (21). Then, for any $k>0$,

$$
\int_{\partial \mathcal{O}_{\varepsilon}}\left|\left(\frac{\partial}{\partial n}+k \lambda\right)\left(u^{0}-\psi^{e i k}(r)\right)\right|^{2} d S<(\varepsilon k)^{2} / 2 .
$$




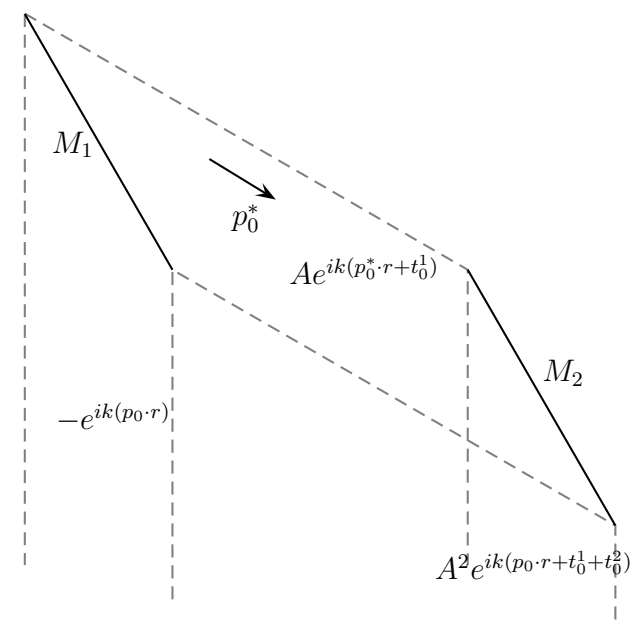

Figure 5: The main term of asymptotics of $\mathcal{L}$

Furthermore, (20) implies that

$$
\int_{\partial \mathcal{O} \backslash \partial \mathcal{O}_{\varepsilon}}\left|\left(\frac{\partial}{\partial n}+k \lambda\right)\left(u^{0}-\psi^{e i k}(r)\right)\right|^{2} d S<C k<(\varepsilon k)^{2} / 2
$$

if $k$ is large enough. Statement of Lemma 11 follows immediately from (36) $)$ (38).

\section{Asymptotic behavior of the total cross sec- tion}

Proof of the second statement of Theorem 1. We begin with the evaluation of the total cross section of the Kirchhoff approximation $u^{0}$ using (23). Consider the boundary $Q$ of a cube such that its faces are parallel to the coordinate planes and which contains the obstacle $\mathcal{O}$. For any small $\delta>0$, we split $Q$ in the following three parts: $Q=Q_{1} \cup Q_{2} \cup Q_{3}$, where $Q_{1}$ is a $\delta$-neighborhood of $Q \cap \widehat{\mathcal{B}}$ (see definition of $\widehat{\mathcal{B}}$ in Lemma 21), $Q_{2}$ is the orthogonal projection of the obstacle $\mathcal{O}$ into the low horizontal side of $Q$ without points already included into $Q_{1}$, and $Q_{3}$ is the remaining part of $Q$.

Note that area $\left|Q_{2}\right|$ of $Q_{2}$ converges to $1 / 2$ as $\delta \rightarrow 0$. Thus, from (21) it follows that for any $\varepsilon>0$ one can choose $\delta$ such that

$$
\frac{1}{k} \int_{Q_{1}}\left|\bar{u}^{0} \frac{\partial u^{0}}{\partial r}\right| d S \leq \frac{\varepsilon}{4} \text { and } 0<\frac{1}{2}-\left|Q_{2}\right| \leq \frac{\varepsilon}{4} .
$$

With $\delta$ fixed, one can choose $k_{0}$ such that

$$
\frac{1}{k} \int_{Q_{3}}\left|\bar{u}^{0} \frac{\partial u^{0}}{\partial r}\right| d S \leq \frac{\varepsilon}{4}, \quad \frac{1}{k}\left|\Im \int_{Q_{2}} \bar{u}^{0} \frac{\partial u^{0}}{\partial r} d S-Z\right| \leq \frac{\varepsilon}{4},
$$


where $Z=\left|-1+A^{2} e^{i k \Delta}\right|^{2}$. The latter relations follow from (20), since (see Fig. 21) $\psi^{e i k}=\Psi^{e i k}-e^{i k z}=0$ on $Q_{3}$, and

$$
\psi^{e i k}=A^{2} e^{i k(z+\Delta)}-e^{i k z} \text { on } Q_{1} .
$$

Inequalities (39), (40) and formula (23) imply the validity of (6) for $u^{0}$. Its validity for $u$ follows from (18). The proof is complete.

\section{Asymptotics of the transport cross section}

The goal of this section is to show that the transport cross section for the obstacle under consideration vanishes as $k \rightarrow \infty$ if $\Im \lambda>0$. The last statement of theorem 1 follows immediately (see the last paragraph of this section) from the first two statements. As the first step, we need to obtain an integral representation for the scattering amplitude of the Kirchhoff approximation. Recall, that $u^{0}=\mathcal{L}+\mathcal{R}$ (see (34)), where $\mathcal{L}$ is given by (35) and $\mathcal{R}$ has a similar form. Denote the scattering amplitude of the functions $u^{0}, \mathcal{L}, \mathcal{R}$ by $u_{\infty}^{0}, \mathcal{L}_{\infty}, \mathcal{R}_{\infty}$, respectively (see (4) where the scattering amplitude is defined for $u$ ). Recall that $M_{1}=\partial O_{l}^{1}, M_{2}=\partial O_{r}^{2}$, and $M_{2}$ is the shift of $M_{1}$; to be more precise, $M_{2}=M_{1}+d p_{0}^{*}, d>0$.

Lemma 4. We have

$$
u_{\infty}^{0}=\mathcal{L}_{\infty}+\mathcal{R}_{\infty}
$$

where

$$
\mathcal{L}_{\infty}=\frac{i k}{4 \pi} \Psi(\theta) \int_{M_{1}} \eta e^{i k\left(p_{0}-\theta\right) \cdot q} d S_{q}
$$

with

$$
\Psi(\theta)=(A-1) n_{p_{0}}-(A+1) n_{\theta}+A e^{i k d\left(1-p_{0}^{*} \cdot \theta\right)}\left((A-1) n_{p_{0}}+(A+1) n_{\theta}\right) .
$$

Function $\mathcal{R}_{\infty}$ is the reflection of $\mathcal{L}_{\infty}$ with respect to the first argument, i.e $\mathcal{R}_{\infty}(\theta)=\mathcal{L}_{\infty}(\bar{\theta})$, where $\bar{\theta}=\bar{\theta}(\theta)=\left(-\theta_{1}, \theta_{2}, \theta_{3}\right)$.

Proof. The validity of (41) follows from (34), so one needs only to find the terms in the right hand side of (41). Scattering amplitude $\mathcal{L}_{\infty}$ can be found from (35):

$$
\begin{aligned}
\mathcal{L}_{\infty} & =-\frac{1}{4 \pi} \int_{M_{1} \cup M_{2}} \eta \frac{\partial}{\partial n_{q}}\left(\Psi^{e i k}(q)\right) e^{-i k \theta \cdot q} d S_{q}-\frac{i k n_{\theta}}{4 \pi} \int_{M_{1} \cup M_{2}} \eta \Psi^{e i k}(q) e^{-i k \theta \cdot q} d S_{q} \\
& =-\frac{1}{4 \pi} \int_{M_{1} \cup M_{2}} \eta\left(\frac{\partial}{\partial n_{q}}\left(\Psi^{e i k}(q)\right)+i k n_{\theta} \Psi^{e i k}(q)\right) e^{-i k \theta \cdot q} d S_{q}, \quad \theta \in S^{2} .
\end{aligned}
$$


We need to insert here the values of the eikonal approximation $\Psi^{\text {eik }}(q)$ on $M_{1}$ and $M_{2}$. Its value and the value of the normal derivative on $M_{1}$ are given by (30) with $\alpha=p_{0}$. In order to find the corresponding values on $M_{2}$ one needs to take into account the following fact which was discussed in section 5 . The wave $\Psi^{e i k}(r)$ shortly before the incident to $M_{2}$ has the form $A e^{i k\left(p_{0}^{*} \cdot r+t_{0}\right)}$, i.e., it differs from the original wave $e^{i k p_{0} \cdot r}$ coming to $M_{1}$ by the choice of the incident direction $\left(p_{0}^{*}\right.$ instead of $\left.p_{0}\right)$ and an extra factor $A e^{i k t_{0}}$. Thus, $\Psi^{e i k}(r)$ and its derivative on $M_{2}$ are given by (30) with $\alpha$ replaced by $p_{0}^{*}$ and the extra factor $A e^{i k t_{0}}$ added in the right-hand sides of (30). Hence, (44) can be rewritten in the form

$$
\begin{gathered}
\mathcal{L}_{\infty}=-\frac{i k}{4 \pi} \int_{M_{1}} \eta\left((-A+1) n_{p_{0}}+(A+1) n_{\theta}\right) e^{i k\left(p_{0}-\theta\right) \cdot q} d S_{q} \\
-\frac{i k A e^{i k t_{0}}}{4 \pi} \int_{M_{2}} \eta\left((-A+1) n_{p_{0}^{*}}+(A+1) n_{\theta}\right) e^{i k\left(p_{0}^{*}-\theta\right) \cdot q} d S_{q} .
\end{gathered}
$$

In order to prove (42), it remains only to rewrite the last integral above as an integral over $M_{1}$ by using the substitution $q \rightarrow q+d p_{0}^{*}$ and the following two facts. Firstly, the normals $n$ on $M_{1}$ and $M_{2}$ have different direction, and therefore, $n_{p_{0}^{*}}, n_{\theta}$ on $M_{2}$ are equal to $n_{p_{0}},-n_{\theta}$ on $M_{1}$, respectively. Secondly, since $p_{0}^{*}=p_{0}-2\left(p_{0} \cdot n\right) n$ and $t_{0}$ is given by (29) where $M=M_{1}$, the exponent in the second integral above (with $q$ replaced by $q+d p_{0}^{*}, q \in M_{1}$ ) can be rewritten as follows

$$
\begin{gathered}
\left.\left(p_{0}^{*}-\theta\right) \cdot\left(q+d p_{0}^{*}\right)\right)=\left(p_{0}^{*}-\theta\right) \cdot q+d\left(1-p_{0}^{*} \cdot \theta\right) \\
=\left(p_{0}-\theta\right) \cdot q-2\left(n \cdot p_{0}\right)(n \cdot q)+d\left(1-p_{0}^{*} \cdot \theta\right)=\left(p_{0}-\theta\right) \cdot q+d\left(1-p_{0}^{*} \cdot \theta\right)-t_{0} .
\end{gathered}
$$

The symmetry between $\mathcal{L}_{\infty}$ and $\mathcal{R}_{\infty}$ can be observed from formula (44) for $\mathcal{L}_{\infty}$, and the corresponding formula for $\mathcal{R}_{\infty}$.

The proof of Lemma 4 is complete.

Theorem 4. If $\Im \lambda>0$ then the transport cross section of the Kirchhoff approximation $u_{0}$ vanishes as $k \rightarrow \infty$. To be precise,

$$
\left.\int_{S^{2}}\left(1-\theta \cdot p_{0}\right)\right)\left|u_{\infty}^{0}(\theta)\right|^{2} d \mu(\theta)=O\left(k^{-\varepsilon}\right), \quad \varepsilon>0, \quad k \rightarrow \infty .
$$

Proof. Denote the integral in (42) by $D_{\infty}(\theta)$; it is equal to the scattering amplitude of the single layer (24). From Lemma 4 it follows that it is enough to prove the statement of the theorem for the part $\mathcal{L}_{\infty}$ of $u_{0}$, i.e. Theorem 4 will be proved as soon as we show that

$$
\int_{S^{2}} \beta_{p_{0}}(\theta)\left|\Psi(\theta) D_{\infty}(\theta)\right|^{2} d S=O\left(k^{-2-\varepsilon}\right), \quad \varepsilon>0 ; \quad \beta_{p_{0}}(\theta)=1-p_{0} \cdot \theta, \quad \Im \lambda>0 .
$$


We do not need the exact form of the functions $\beta_{p_{0}}, \Psi$, but only some estimates valid for those functions. Namely,

$$
\begin{gathered}
0 \leq \beta_{p_{0}}(\theta) \leq C\left|\theta-p_{0}\right|^{2}, \quad \theta \in S^{2}, \\
|\Psi(\theta)| \leq C, \quad|\Psi(\theta)| \leq C\left(\left|\theta-p_{0}^{*}\right|+k\left|\theta-p_{0}^{*}\right|^{2}\right), \quad \theta \in S^{2},
\end{gathered}
$$

In fact, $\beta$ is a real valued infinitely smooth function on $S^{2}$ with the minimum value at the point $\theta=p_{0}$. Thus, the validity of the quadratic estimate (46) is obvious. In order to justify (47), we represent $\Psi$ in the form $\Psi(\theta)=\Psi_{1}(\theta)+\Psi_{2}(\theta)$ where $\Psi_{1}(\theta)$ is given by (43) with the exponential factor in that formula omitted, and

$$
\Psi_{2}(\theta)=\Psi(\theta)-\Psi_{1}(\theta)=A\left(e^{i k d\left(1-p_{0}^{*} \cdot \theta\right)}-1\right)\left((A-1) n_{p_{0}}+(A+1) n_{\theta}\right) .
$$

Since $n_{p_{0}^{*}}=-n_{p_{0}}$, function $\Psi_{1}(\theta)$ vanishes at $\theta=p_{0}^{*}$. This function is infinitely smooth, complex-valued and $k$-independent. Thus, $\left|\Psi_{1}(\theta)\right| \leq C \mid \theta-$ $p_{0}^{*} \mid$. Further,

$$
\left|\Psi_{2}(\theta)\right| \leq C\left|e^{i k \beta_{p_{0}^{*}}(\theta)}-1\right| \leq C k\left|\theta-p_{0}^{*}\right|^{2} .
$$

The latter inequality follows immediately from (46). Hence, the validity of both (46) and (47) are justified.

For any vector $u \in S^{2}$ denote by $\widetilde{u}$ the orthogonal projection of $u$ on the plane $P$ containing $M_{1}$. Vectors $p_{0}, p_{0}^{*}$ have the same projections $\widetilde{p_{0}}=\widetilde{p_{0}^{*}}$. Let $B_{\widetilde{p_{0}}}\left(k^{-\gamma}\right)$ be the disk in $P$ of the radius $k^{-\gamma}, \frac{2}{3}<\gamma<\frac{3}{4}$, centered at $\widetilde{p_{0}}$. Denote by $\Omega_{1}, \Omega_{2}$ small neighborhoods on $S^{2}$ of the points $p_{0}, p_{0}^{*}$, respectively, whose projections on $P$ coincide with $B_{\widetilde{p_{0}}}\left(k^{-\gamma}\right)$. Then

$$
\left|\widetilde{\theta}-\widetilde{p_{0}}\right|>k^{-\gamma}>0 \text { when } \theta \in \Omega_{3}=S^{2} \backslash\left(\Omega_{1} \cup \Omega_{2}\right) .
$$

Denote by $\widetilde{\Delta}=\widetilde{\Delta}_{p}$ the two-dimensional Laplace operator in the plane $P$. For any $q, q_{0} \in M_{1}$ and $p=q-q_{0} \in P$, we have

$$
\widetilde{\Delta}_{p} e^{i k\left(p_{0}-\theta\right) \cdot q}=\widetilde{\Delta}_{p} e^{i k\left[\left(p_{0}-\theta\right) \cdot q_{0}+\left(\widetilde{p_{0}}-\widetilde{\theta}\right) \cdot p\right]}=-k^{2}\left|\widetilde{p_{0}}-\widetilde{\theta}\right|^{2} e^{i k\left(p_{0}-\theta\right) \cdot q} .
$$

Hence, from (48) and (15) it follows that for any $m$

$$
\begin{gathered}
\mathcal{D}_{\infty}(\theta)=\frac{1}{(i k)^{2 m}\left|\widetilde{p_{0}}-\widetilde{\theta}\right|^{2 m}} \int_{M_{1}} \eta \widetilde{\Delta}^{m} e^{i k\left(p_{0}-\theta\right) \cdot q} d S_{q} \\
=\frac{1}{(i k)^{2 m}\left|\widetilde{p_{0}}-\widetilde{\theta}\right|^{2 m}} \int_{M_{1}}\left(\widetilde{\Delta}^{m} \eta\right) e^{i k\left(p_{0}-\theta\right) \cdot q} d S_{q}=O\left(k^{-2 m\left(\frac{3}{4}-\gamma\right)}\right), \quad \theta \in \Omega_{3} .
\end{gathered}
$$


Since $\gamma<3 / 4$ and $m>0$ is arbitrary, it remains to show that

$$
I_{i}:=k^{2} \int_{\Omega_{i}} \beta_{p_{0}}(\theta)\left|\Psi(\theta) D_{\infty}(\theta)\right|^{2} d S=O\left(k^{-\varepsilon}\right), \quad i=1,2, \quad \Im \lambda>0 .
$$

Let us prove (49) for $i=2$. The case $i=1$ can be treated similarly (in fact, the latter case is simpler with the reference to (46) instead of (47)). We use Euclidean coordinates on $P$ as local coordinates on $\Omega_{2}: \theta=\theta(\widetilde{\theta})$. Since $p_{0}, p_{0}^{*}$ are transversal to $P$, the Jacobian $J(\theta)=\frac{d S}{d S_{\widetilde{\theta}}}=1 / \sqrt{\left(1-|\widetilde{\theta}|^{2}\right)}$ is bounded when $\theta \in \Omega_{1}$, and

$$
\left|\widetilde{\theta}-\widetilde{p_{0}^{*}}\right| \leq\left|\theta-p_{0}^{*}\right| \leq C\left|\widetilde{\theta}-\widetilde{p_{0}^{*}}\right|, \quad \theta \in \Omega_{1} .
$$

From here, (47) and the boundedness of $\beta(\theta)$ and $\left|D_{\infty}(\theta)\right|$ it follows that

$$
\begin{array}{r}
I_{2} \leq C k^{2} \int_{B_{p_{0}}\left(k^{-\gamma}\right)}|\Psi(\theta)|^{2} d S_{\widetilde{\theta}} \leq C \int_{B_{p_{0}}\left(k^{-\gamma}\right)}\left(k^{2}\left|\widetilde{\theta}-\widetilde{p_{0}^{*}}\right|^{2}+k^{4}\left|\widetilde{\theta}-{\widetilde{p_{0}^{*}}}^{*}\right|^{4}\right) d S_{\widetilde{\theta}} \\
\leq 2 \pi C \int_{0}^{k^{-\gamma}}\left(k^{2} \sigma^{3}+k^{4} \sigma^{5}\right) d \sigma=O\left(k^{-\varepsilon}\right), \quad \varepsilon=6 \gamma-4>0 .
\end{array}
$$

The proof of Theorem 4 is complete.

Proof of the first and the last statements of Theorem 1. The first statement of Theorem 1 follows immediately from (19) and Theorem 4. Let us prove the last statement. It follows from the first statement that for any neighborhood $S_{\delta} \subset S^{2}$ of the point $p_{0}$, we have $\int_{S^{2} \backslash S_{\delta}}\left|u_{\infty}\right|^{2} d S \rightarrow 0, k \rightarrow \infty$. Then from the second statement we obtain that

$$
\int_{S_{\delta}}\left|u_{\infty}\right|^{2} d S \rightarrow \frac{1}{2}\left|-1+A^{2} e^{i k \Delta}\right|^{2}, \quad k \rightarrow \infty .
$$

Obviously, the latter two relations imply (7).

\section{Real impedance}

Proof of Theorem 2, Let us fix $\lambda_{0} \in \mathbb{R}$. We will need the optical theorem for $\lambda \in \mathbb{R}$ :

$$
\sigma_{\lambda}(k)=(4 \pi / k) \Im u_{\infty}\left(p_{0}\right), \quad \lambda \in \mathbb{R} .
$$

Note that this theorem concerns the exact solution of the scattering problem, i.e., it is valid for $u_{\infty}$ but not for the far field given by the Kirchhoff 
approximation $u_{\infty}^{0}$. Hence, asymptotic behavior of $\Im u_{\infty}^{0}\left(p_{0}\right)$ can not be derived from the known asymptotics of $\left\|u_{\infty}^{0}\right\|$. We therefore calculate it independently. From lemma 4 we have

$$
u_{\infty}^{0}\left(p_{0}\right)=\frac{i k}{2 \pi} \Psi\left(p_{0}\right) \int_{M} \eta(q) d S_{q}=\frac{i k}{\pi} n_{p_{0}}\left(-1+A^{2} e^{i k d\left(1-p_{0} \cdot p_{0}^{*}\right)}\right) \int_{M} \eta(q) d S_{q} .
$$

Note that $d=\left|A^{\prime} B^{\prime \prime}\right|$ (see Fig. 2) is the hypotenuse of the triangle $A^{\prime} B^{\prime \prime} H$. Thus $d\left(1-p_{0} \cdot p_{0}^{*}\right)=d-\left|B^{\prime \prime} H\right|=\Delta$, and

$$
u_{\infty}^{0}\left(p_{0}\right)=\frac{i k}{4 \pi}\left(-1+A^{2} e^{i k \Delta}\right)(1+o(1)), \quad k \rightarrow \infty .
$$

Hence, if $k=k_{n}$ defined by (8) and $\lambda$ is real, then on noting that $A(\lambda)$ does not depend on $k$ and $|A|=1$ for real $\lambda$,

$$
u_{\infty}^{0}\left(p_{0}\right)=\frac{i k_{n}}{4 \pi}\left(-1+e^{2 i\left(\operatorname{Arg}(A(\lambda))-\operatorname{Arg}\left(A\left(\lambda_{0}\right)\right)\right.}\right)(1+o(1)), \quad k_{n} \rightarrow \infty, \quad \Im \lambda=0 .
$$

Our next goal is to estimate the difference $f_{\lambda}\left(p_{0}\right):=u_{\infty}^{0}\left(p_{0}\right)-u_{\infty}\left(p_{0}\right)$ for complex $\lambda, \Im \lambda>0$. First of all note that the relation (22) for the scattering amplitude is valid also for $u$ and $v=u^{0}-u$. Thus,

$$
\left|f_{\lambda}\left(p_{0}\right)\right| \leq\left\|v_{n}\right\|_{L_{2}(\partial \mathcal{O})}+\|k v\|_{L_{2}(\partial \mathcal{O})} .
$$

Denote by $\Lambda$ the set $\Lambda=\left\{\lambda:\left|\lambda-\lambda_{0}\right| \leq 1, \Im \lambda>0\right\}$. Statements of Lemma 1 and Theorem 3 are valid uniformly in $\lambda \in \Lambda$. Together with the estimate above this implies that

$$
\left|f_{\lambda}\left(p_{0}\right)\right| \leq \frac{o(k)}{\Im \lambda}, \quad k \rightarrow \infty, \quad \lambda \in \Lambda
$$

where $o(k)$ is uniform in $\lambda \in \Lambda$.

Let $\varepsilon>0$. Note that $\min _{\lambda \in\left(\lambda_{0}-\varepsilon, \lambda_{0}+\varepsilon\right)}\left(\lambda-\left(\lambda_{0}-2 \varepsilon\right)\right)\left(\left(\lambda_{0}+2 \varepsilon\right)-\lambda\right)=3 \varepsilon^{2}$. Thus,

$$
\Phi(\lambda):=\frac{\left(\lambda-\left(\lambda_{0}-2 \varepsilon\right)\right)\left(\left(\lambda_{0}+2 \varepsilon\right)-\lambda\right)}{3 \varepsilon^{2}} \geq 1, \quad \lambda \in\left[\lambda_{0}-\varepsilon, \lambda_{0}+\varepsilon\right] .
$$

Now we are ready to estimate the average of the function (501). Using (53) and the positiveness of the function (50) for real $\lambda$ and $\Phi(\lambda)$ for $\lambda \in$ $\left(\lambda_{0}-2 \varepsilon, \lambda_{0}+2 \varepsilon\right)$, we get

$$
\frac{1}{k_{n}} \int_{\lambda_{0}-\varepsilon}^{\lambda_{0}+\varepsilon} \Im u_{\infty}\left(p_{0}\right) d \lambda \leq \frac{1}{k_{n}} \int_{\lambda_{0}-\varepsilon}^{\lambda_{0}+\varepsilon} \Phi(\lambda) \Im u_{\infty}\left(p_{0}\right) d \lambda \leq \frac{1}{k_{n}} \int_{\lambda_{0}-2 \varepsilon}^{\lambda_{0}+2 \varepsilon} \Phi(\lambda) \Im u_{\infty}\left(p_{0}\right) d \lambda
$$




$$
=\frac{1}{k_{n}} \int_{\lambda_{0}-2 \varepsilon}^{\lambda_{0}+2 \varepsilon} \Phi(\lambda) \Im u_{\infty}^{0}\left(p_{0}\right) d \lambda-\frac{1}{k_{n}} \int_{\lambda_{0}-2 \varepsilon}^{\lambda_{0}+2 \varepsilon} \Phi(\lambda) \Im f_{\lambda}\left(p_{0}\right) d \lambda .
$$

From (51), the integrand of the first integral in the right hand side of (54) does not exceed $C \varepsilon$, i.e., the first term does not exceed $C \varepsilon^{2}$, where the constant $C$ is independent of $\varepsilon$ and $k$.

Let us study the second part in (54). Since $\Phi(\lambda)$ is a real-valued function for real $\lambda$, we have

$$
\int_{\lambda_{0}-2 \varepsilon}^{\lambda_{0}+2 \varepsilon} \Phi(\lambda) \Im f_{\lambda}\left(\theta_{0}\right) d \lambda=\Im \int_{\lambda_{0}-2 \varepsilon}^{\lambda_{0}+2 \varepsilon} \Phi(\lambda) f_{\lambda}\left(\theta_{0}\right) d \lambda .
$$

One can easily see that the Kirchhoff approximation $u^{0}$ defined by (14) is analytic in $\lambda$. Then from (22) it follows that $u_{\infty}^{0}$ is analytic in $\lambda$. The function $u_{\infty}$ is also analytic in $\lambda, \Im \lambda \geq 0$, (see eg [4], [5]). Thus, $f_{\lambda}\left(\theta_{0}\right)$ is analytic when $\Im \lambda \geq 0$, and the contour of integration in (55) can be replaced by the contour $\Gamma=\Gamma_{1} \cup \Gamma_{2} \cup \Gamma_{3}$ defined in Fig. 6.

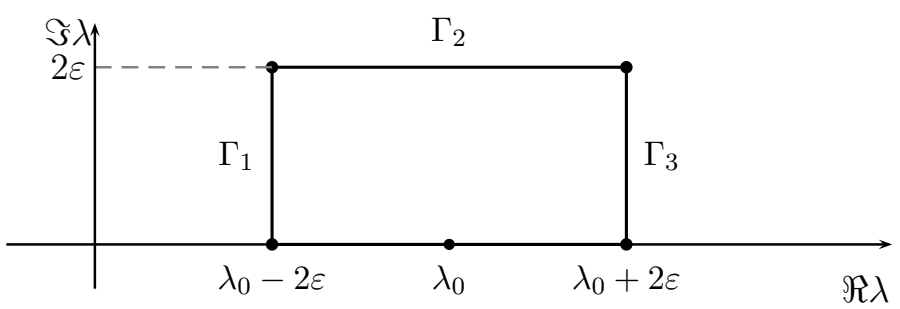

Figure 6: Contour $\Gamma$

From here and (52) it follows that

$$
\left|\int_{\lambda_{0}-2 \varepsilon}^{\lambda_{0}+2 \varepsilon} \Phi(\lambda) f_{\lambda}\left(\theta_{0}\right) d \lambda\right|=\left|\int_{\Gamma} \Phi(\lambda) f_{\lambda}\left(\theta_{0}\right) d \lambda\right| \leq o(k) \max _{\Gamma} \frac{|\Phi(\lambda)|}{\Im \lambda}|\Gamma| .
$$

One can show that $\max _{\Gamma} \frac{|\Phi(\lambda)|}{\Im \lambda} \leq \frac{C}{\varepsilon}$. Since $|\Gamma|=8 \varepsilon$, we obtain the following relation which holds for the second term in the right-hand side of (54). There exists $\varepsilon_{0}>0$ such that

$$
\delta_{n}:=\max _{0<\varepsilon<\varepsilon_{0}} \frac{1}{k_{n}}\left|\int_{\lambda_{0}-2 \varepsilon}^{\lambda_{0}+2 \varepsilon} \Phi(\lambda) f_{\lambda}\left(\theta_{0}\right) d \lambda\right| \rightarrow 0, \quad k_{n} \rightarrow \infty .
$$

Now from (54) and (50) we get that, for $\varepsilon \in\left(0, \varepsilon_{0}\right)$,

$$
\frac{1}{2 \varepsilon} \int_{\lambda_{0}-\varepsilon}^{\lambda_{0}+\varepsilon} \sigma_{\lambda}\left(k_{n}\right) d \lambda \leq C \varepsilon+\frac{\delta_{n}}{\varepsilon}, \quad k_{n} \rightarrow \infty
$$

The choice $\varepsilon_{n}=\sqrt{\delta_{n}}$ implies the statement of Theorem 2. The Consequence from the theorem follows if $\lambda_{0}$ is chosen to be the minimum value of $\sigma_{\lambda}\left(k_{n}\right)$ on the interval $\lambda_{0}-\varepsilon_{n} \leq \lambda \leq \lambda_{0}+\varepsilon_{n}$. 


\section{Appendix I}

Here we prove Lemma 3. The proof is a direct consequence of formula (31) and lemmas 5 and 6 below concerning the asymptotic behavior of the simple and double layers as $k \rightarrow \infty, r$ is bounded. These asymptotics are different in the shadow zone, the reflected zone and in the complementary region.

We show that on $M$ and in shadow zone we have

$$
D(r)=\frac{2 \pi}{i k n_{\alpha}} e^{i k(\alpha, r)}+O\left(k^{-3 / 2}\right), \quad r \in M \cup S(\alpha), \quad k \rightarrow \infty .
$$

If $r$ belongs to the reflected zone, then

$$
D(r)=\frac{2 \pi}{i k n_{\alpha}} e^{i k t_{0}} e^{i k\left(\alpha^{*} \cdot r\right)}+O\left(k^{-3 / 2}\right), \quad r \in R(\alpha), \quad k \rightarrow \infty,
$$

where constant $t_{0}=t_{0}(\alpha, M)$ defined from the continuity on $M$, i.e.,

$$
e^{i k t_{0}} e^{i k\left(\alpha^{*} \cdot r\right)}=e^{i k(\alpha \cdot r)}, \quad r \in M .
$$

If $r$ is in the complement to those zones, then

$$
D(r)=O\left(k^{-3 / 2}\right), \quad k \rightarrow \infty
$$

However, these asymptotics are not uniform, and exact statement is as follows.

Lemma 5. 1. Expansion (56) is valid uniformly on any compact subset of $M$ and on any compact set strictly inside of the shadow zone. Expansion (57) is valid uniformly on any compact set strictly inside of the reflected zone.

2. Estimate (59) is valid uniformly on any compact set which does not have common points with the closure of the shadow or illuminated zones.

3. The above expansions on compact sets strictly inside $S(\alpha)$ or $R(\alpha)$, or strictly outside of these zones can be differentiated any number of times with the remainders multiplied by $k$ after each differentiation.

4. Derivatives of $D(r)$ of any order in directions tangential to $M$ are continuous on $M$, and the expansion (56) on $M$ can be differentiated any number of times in those directions (with the corresponding increase of the order of the remainders). The normal derivative of $D(r)$ has a jump on $M$, and

$$
\frac{\partial}{\partial n} D(r)=-2 \pi \eta e^{i k(\alpha, r)}, \quad r \in M,
$$

where the left hand side is defined according to (25) 
5. The following uniform bound is valid on any bounded region of $R^{3}$

$$
\begin{gathered}
|D(r)| \leq \frac{C\left(R_{0}\right)}{k}, \quad|r|<R_{0}, k \rightarrow \infty, \\
\left|P_{m} D(r)\right| \leq C\left(R_{0}\right) k^{m-1}, \quad r \notin \bar{M},|r|<R_{0}, k \rightarrow \infty,
\end{gathered}
$$

where $P_{m}$ is an arbitrary partial derivative of order $m$.

Proof. First, we discuss the smoothness of $D(r)$ on $M$ (obviously $D \in$ $C^{\infty}$ when $\left.r \notin \bar{M}\right)$. Since the integrand in (24) has a weak singularity, $D(r)$ is continuous on $\bar{M}$. Further, let $r \notin \bar{M}, q \in M$. For any unit vector $v$ orthogonal to $n$ (i.e. $v$ is in the plane containing $M$ ), we have $\frac{\partial}{\partial v}|r-q|=$ $-\frac{\partial}{\partial v_{q}}|r-q|$ where $\frac{\partial}{\partial v}, \frac{\partial}{\partial v_{q}}$ are derivatives in the direction of the vector $v$ with respect to $r$ and $q$, respectively. Thus, after integration by parts we get

$$
\begin{aligned}
& \frac{\partial}{\partial v} D(r)=\int_{M} \eta e^{i k(\alpha, q)} \frac{\frac{\partial}{\partial v} e^{i k|r-q|}}{|r-q|} d S_{q}-\int_{M} \eta e^{i k(\alpha, q)} e^{i k|r-q|} \frac{\partial}{\partial v_{q}}\left[\frac{1}{|r-q|}\right] d S_{q} \\
= & \int_{M} \frac{\eta}{|r-q|}\left(\frac{\partial}{\partial v}+\frac{\partial}{\partial v_{q}}\right) e^{i k((\alpha, q)+|r-q|)} d S_{q}+H^{(v)}=i k(\alpha, v) D(r)+H^{(v)},
\end{aligned}
$$

where $H^{(v)}=\int_{M}\left(\frac{\partial}{\partial v_{q}} \eta\right) \frac{e^{i k((\alpha, q)+|r-q|)}}{|r-q|} d S_{q}$. Obviously $H^{(v)}$ is continuous, and so is $\frac{\partial}{\partial v} D(r)$. If the vector $u$ is also orthogonal to $n$, then the same arguments imply

$$
\begin{gathered}
\frac{\partial^{2}}{\partial u \partial v} D(r)=- \\
k^{2}(\alpha, u)(\alpha, v) D(r)+i k(\alpha, v) H^{u}+i k(\alpha, u) H^{v} \\
+\int_{M} \frac{\partial^{2} \eta}{\partial u_{q} \partial v_{q}} \frac{e^{i k((\alpha, q)+|r-q|)}}{|r-q|} d S_{q} .
\end{gathered}
$$

This provides continuity of second derivatives. The higher order derivatives can be treated similarly.

The normal derivative of a single layer has a jump on the surface of integration. This fact and the limiting values of the normal derivatives can be found in the many textbooks on partial differential equations. In our case the limit (60) of the normal derivative is proportional to the density and does not contain an integral term since the surface of integration is flat. Formula (60) can also be treated as a simple exercise in the distribution theory.

Our next goal is to find the asymptotics of $D(r), k \rightarrow \infty$, on a compact subset $K \subset M$. Note that the integrand in (24) is not smooth in this case, and the stationary phase method cannot be applied. Let us introduce polar coordinates $(\rho, \phi)$ on the plane $P$ with the center at the point $r$ and the 
polar angle being zero along of the ray $\tau$ whose direction coincides with the direction of the projection $\widehat{\alpha}$ of the vector $\alpha$ on $P$. If $\alpha$ is orthogonal to $P$ (i.e. $\widehat{\alpha}=0$ ), then $\tau$ can be chosen arbitrarily. Polygon $\partial M$ in polar coordinates has the form $\rho=\rho(\phi, r), 0 \leq \phi<2 \pi$, where $\rho$ as a function of $\phi$ is continuous, $2 \pi$-periodic and analytic for all the values of $\phi$ except those which correspond to the vertices of $\partial M$. We write the integral $D(r)$ in polar coordinates and integrate by parts in $\rho$. When $r \in K \subset M$ and $k$ is large enough so that $\eta=1$ on $K$, we get

$$
\begin{gathered}
D(r)=e^{i k(\alpha, r)} \int_{0}^{2 \pi} \int_{0}^{\rho(\phi, r)} \eta e^{i k \rho(1+|\widehat{\alpha}| \cos \phi)} d \rho d \phi \\
=\frac{e^{i k(\alpha, r)}}{i k}\left(\int_{0}^{2 \pi} \frac{-1}{1+|\widehat{\alpha}| \cos \phi} d \phi-\int_{0}^{2 \pi} \int_{0}^{\rho(\phi, r)} \eta_{\rho} \frac{e^{i k \rho(\phi, r)(1+|\widehat{\alpha}| \cos \phi)}}{1+|\widehat{\alpha}| \cos \phi} d \rho d \phi\right),
\end{gathered}
$$

Since vector $\alpha$ is transversal to $P,|\widehat{\alpha}|<1$, and $\int_{0}^{2 \pi} \frac{d \phi}{1+|\widehat{\alpha}| \cos \phi}=\frac{2 \pi}{\sqrt{1-|\widehat{\alpha}|^{2}}}=\frac{-2 \pi}{n_{\alpha}}$, formula (65) implies

$$
D(r)=\frac{1}{i k} e^{i k(\alpha, r)}\left(\frac{2 \pi}{n_{\alpha}}-\int_{0}^{2 \pi} \int_{0}^{\rho(\phi, r)} \eta_{\rho} \frac{e^{i k \rho(\phi, r)(1+|\widehat{\alpha}| \cos \phi)}}{1+|\widehat{\alpha}| \cos \phi} d \rho d \phi\right), \quad r \in K \subset M .
$$

The interior integral, above after integration by parts $m$ times, takes the form

$$
\left(\frac{-1}{i k}\right)^{m} \int_{0}^{\rho(\phi, r)} \frac{\partial^{m+1} \eta}{(\partial \rho)^{m+1}} \frac{e^{i k \rho(\phi, r)(1+|\widehat{\alpha}| \cos \phi)}}{(1+|\widehat{\alpha}| \cos \phi)^{m+1}} d \rho=O\left(k^{(1-3 m) / 4}\right), \quad k \rightarrow \infty .
$$

The latter relation follows from (15). Hence, for $r \in K \subset M$, (66) implies (56).

The tangential derivatives of $D(r)$ can be evaluated using (63), (64) and similar formulas for the derivatives of higher order. One only needs to note that functions similar to $H^{(v)}$ have order $O\left(k^{-\infty}\right), k \rightarrow \infty$, i.e., they decay at infinity faster than any power of $k$. This can be justified precisely as the similar statement above for the integral in (66). Thus, the asymptotics (56) on $M$ can be differentiated in tangential directions.

We now consider the asymptotics of $D(r)$ and its derivatives on compact sets outside of the boundaries of the shadow and reflected zones. If $r \notin \bar{M}$, the integrand in (24) is smooth, and the stationary phase method can be applied. Let $F=F(q)$ be the phase function in (24). Since $\nabla_{q} F=\alpha-\frac{r-q}{|r-q|}$, the stationary phase points are defined by the equation

$$
\alpha-\frac{r-q}{|r-q|}=c n, \text { where } c \text { is a constant, } q \in M .
$$


Both vectors on the left are unit vectors, and therefore, (67) holds if and only if $r-q=c_{1} \alpha$ or $r-q=c_{2} \alpha^{*}$, where $c_{i}=|r-q| \geq 0, q \in M$. This is possible only if $r$ is in the shadow or reflected zone, respectively. The stationary point is on $\partial M$ if and only if $r$ belongs to the lateral boundary of these zones. If $r$ belongs to a compact set whose intersection with the boundaries of those zones is empty, the stationary phase point is strictly inside $M$ or outside of $\bar{M}$. Thus, the stationary phase method implies the uniform validity of the expansions (56), (57), (59) for $D$ and its derivatives on these compact sets.

Since the amplitude factor $\eta(k, q) /|r-q|$ in the integrand (24) depends on $k$, we will justify the latter statement a little more rigorously. Consider a compact set $J$ strictly inside of the reflected zone. Let $J^{\prime}$ be the projection of $J$ into $M$ parallel to the vector $\alpha^{*}$. Then $J^{\prime}$ is located strictly inside $M$, and the stationary phase point belongs to $J^{\prime}$ when $r \in M$. Let $\varsigma=\varsigma(r) \in C^{\infty}(M)$ be an infinitely smooth function which is equal to one on $J^{\prime}$ and zero in some neighborhood of $\partial M$. Let $k$ be so large that $\eta=0$ on the support of $\varsigma$. We write $D(r)$ as the sum $D_{1}(r)+D_{2}(r)$ where $D_{1}, D_{2}$ are given by (24) with additional factors $\eta, 1-\eta$ in the integrand, respectively. The stationary phase method can be applied to $D_{1}$, since the amplitude factor for that integral does not depend on $k$. In order to complete the proof of (57) for $r \in J$ it remains to show that $D_{2}=O\left(k^{-\infty}\right), k \rightarrow \infty$. If $f(k, q)=\eta(1-\varsigma) /|r-q|$, then

$D_{2}=\int_{M} f e^{i k F} d S_{q}=\int_{M} \frac{f}{|\nabla F|^{2}}(\nabla F \cdot \nabla F) e^{i k F} d S_{q}=-\frac{1}{i k} \int_{M} \nabla\left(\frac{f \nabla F}{|\nabla F|^{2}}\right) e^{i k F} d S_{q}$

This integration by parts can be repeated as many times as we please. This combined with (15) provides the estimate for $D_{2}$. Thus, (57) for $r \in J$ is proved. Estimates for the compact sets in the shadow zone and outside of the shadow and reflected zones are treated similarly.

It remains to prove the last statement of Lemma 5. We begin with the proof of (61). The proof will be based on the same arguments which were used above in order to justify the asymptotics of $D(r)$ on $M$. Let us assume first that $r$ belongs to the reflected zone, i.e. $r=q_{0}+t \alpha^{*}, q_{0} \in M, t>0$. We introduce polar coordinates $(\rho, \phi)$ on the plane $P$ with the origin at $q_{0}$ and the polar angle defined in the third paragraph of the proof (where polar coordinates were used). Then similar to the first equality (65), we have

$$
D(r)=e^{i k\left(\alpha, q_{0}\right)} \int_{0}^{2 \pi} \int_{0}^{\rho\left(\phi, q_{0}\right)} \frac{\eta e^{i k f(\rho, t, \phi)}}{g(\rho, t, \phi)} \rho d \rho d \phi,
$$

where $f=\rho|\widehat{\alpha}| \cos \phi+\sqrt{t^{2}-2 t|\widehat{\alpha}| \rho \cos \phi+\rho^{2}}, \quad g=\sqrt{t^{2}-2 t|\widehat{\alpha}| \rho \cos \phi+\rho^{2}}$.

For any function $u(\rho, t, \phi)$, denote by $u^{\prime}$ its derivative with respect to $\rho$. Let $h(\rho, t, \phi)=f^{\prime}(\rho, t, \phi) / \rho$. Since $|\widehat{\alpha}|<1$, one can easily check that 
$h(\rho, 1, \phi) \in C^{\infty}, h(\rho, 1, \phi) \neq 0$ and $0<c_{1} \rho^{-1}<h<c_{2} \rho^{-1}, \rho \rightarrow \infty$. Moreover, $1 /|h g|,\left|(h g)^{\prime}\right| \leq C<\infty$ when $t=1, \rho>0$. Then from the homogeneity of $f$ and $g$ with respect to $(\rho, t)$ it follows that the same estimates (with the same constant $C$ ) hold for all $t>0$ :

$$
\frac{1}{|h g|},\left|(h g)^{\prime}\right| \leq C<\infty, \quad t, \rho>0 .
$$

Using integration by parts, we get:

$$
\begin{aligned}
D(r)= & e^{i k\left(\alpha, q_{0}\right)} \int_{0}^{2 \pi} \int_{0}^{\rho\left(\phi, q_{0}\right)} \frac{\eta}{h(\rho, t, \phi) g(\rho, t, \phi)} f^{\prime}(\rho, t, \phi) e^{i k f(\rho, t, \phi)} d \rho d \phi \\
& =\left.(i k)^{-1} e^{i k\left(\alpha, q_{0}\right)} \int_{0}^{2 \pi}\left[\frac{\eta}{h(\rho, t, \phi) g(\rho, t, \phi)} e^{i k f(\rho, t, \phi)}\right]\right|_{\rho=0} d \phi \\
& -(i k)^{-1} e^{i k\left(\alpha, q_{0}\right)} \int_{0}^{2 \pi} \int_{0}^{\rho\left(\phi, q_{0}\right)}\left(\frac{\eta^{\prime}}{h g}+\eta\left(\frac{1}{h g}\right)^{\prime}\right) e^{i k f(\rho, t, \phi)} d \rho d \phi .
\end{aligned}
$$

We now split the last term into $I_{1}+I_{2}$ with factors $\eta^{\prime}$ and $\eta$ in the integrands, respectively. Formulas (69) justify (61) for the first term on the right hand side of (70I) and for $I_{2}$. They also lead to the estimate $I_{1}<C k^{-1} \int_{M}\left|\eta^{\prime}\right| d S_{q}$ which implies (61) for $I_{1}$, since $\left|\eta^{\prime}\right|=O\left(k^{1 / 4}\right)$ and the support of $\eta^{\prime}$ has measure of order $O\left(k^{-1 / 4}\right)$. The proof of (61) when $r$ is in the reflected zone is complete.

The same arguments are valid if $r$ in the same half space bounded by $P$, but does not belong to the closure of the reflected zone. Then $q_{0}$ does not belong to $M$. The limits of integration in (68) will be different, namely, $\phi \in I, \rho_{1}\left(\phi, q_{0}\right)<\rho<\rho_{1}\left(\phi, q_{0}\right)$. Here $\rho_{1}, \rho_{2}$ are values of $\rho$ where the ray from the origin with the polar angle $\phi$ intersects $\partial M$, and $I$ is the set of values of the angle for which such an intersection is not empty. This leads to (70) where the first term on the right has to be omitted and the limits of integration in the second term have to be changed. Obviously, estimate (61) is still valid. In order to obtain (61) when $r$ is in another half space, one needs only to replaced $\alpha^{*}$ by $\alpha$.

Let us establish the validity of (62). We begin with the case of $P_{m}=D_{u}$, i.e. with the estimate of a tangential derivative of the first order. The desirable estimate follows from (63): the first term in the right-hand side of (63) satisfies (62) due to (61), and the second term has an even more refined estimate. In fact, function $H^{(u)}$ has the same form as $D(r)$, but with $\eta$ replaced by its derivative. Thus the arguments justifying (62) for $D(r)$ will provide the same estimate for $H^{(u)}$ with an extra factor $k^{1 / 4}$ in the righthand side due to (15). Tangential derivatives of $D(r)$ of higher order can be obtained similarly. 
We now estimate $\frac{\partial}{\partial n} D(r)$. Let $r_{0}$ be the orthogonal projection of $r$ onto the plane $P$, i.e. $r=r_{0}+n t, r_{0} \in P, 0 \neq t \in \mathbb{R}$. Then $\frac{\partial}{\partial n} D(r)=I_{1}+i k I_{2}$, where

$$
I_{1}=\int_{M} \eta \frac{-t}{|r-q|^{3}} e^{i k((\alpha \cdot q)+|r-q|)} d S_{q}+\int_{M} \eta \frac{t}{|r-q|^{2}} e^{i k((\alpha \cdot q)+|r-q|)} d S_{q} .
$$

The second factor differs from $D(r)$ only by an extra factor $\frac{t}{|r-q|}$ in the integrand. This factor is bounded and homogeneous in $(r, q)$ of zero order. Thus, the proof of (61) for $D$ (see (68)-(170)) can be repeated for $I_{2}$, i.e. $\left|I_{2}\right|<C / k, k \rightarrow \infty$. $I_{1}$ can be estimated very easily: if $r_{0}^{2}+t^{2}<R_{0}^{2}$, ( $|r|$ is bounded), then by using polar coordinates centered at $r_{0}$ we obtain

$$
\left|I_{1}\right| \leq C \int_{\left|q-r_{0}\right|<c} \frac{|t|}{\left(\left(r_{0}-q\right)^{2}+t^{2}\right)^{3 / 2}} d S_{q} \leq 2 \pi C \int_{0}^{c} \sigma\left(\frac{|t|}{\left(\sigma^{2}+t^{2}\right)^{3 / 2}} \leq C_{1},\right.
$$

i.e. (62) holds for $\frac{\partial}{\partial n} D(r)$.

If $P_{m}$ contains differentiation of order $m-1$ in tangential directions and the derivative of the first order in the normal to $M$ direction, then (62) can be justified by a combination of the arguments used to prove (62) for tangential derivatives and for $\frac{\partial}{\partial n} D(r)$ : one needs to start with (63) or similar formula for the tangential derivatives of higher order, then apply $\partial / \partial n$ and repeat the arguments used to estimate $\frac{\partial}{\partial n} D$. Finally, note that $\left(\triangle+k^{2}\right) D(r)=0$ when $r \notin \bar{M}$. Thus

$$
\frac{\partial^{2}}{\partial n^{2}} D(r)=\left(\Delta_{M}+k^{2}\right) D(r), \quad r \notin M,
$$

where $\Delta_{M}$ is the two-dimensional Laplacian on $M$. Hence $P_{m} D(r)$ for arbitrary $P_{m}$ can be expressed through derivatives of $D$ containing differentiation in the normal direction of at most first order. This proves (62) for arbitrary $P_{m}$.

The proof of Lemma 5 is complete.

The double layer potential $N(r)=N_{\alpha, M}(r)$ is given by (26), (27). Obviously, since $M$ is flat, $N(r)=-\frac{\partial}{\partial n} D(r)$. Thus, the next lemma about the properties of $N(r)$ is a direct consequence of Lemma 5 and (171).

Lemma 6. 1. If $r \in M$ and $k \rightarrow \infty$, then

$$
N(r)=2 \pi e^{i k(\alpha \cdot r)}, \quad \frac{\partial}{\partial n} N(r)=-2 \pi i k(n \cdot \alpha) e^{i k(\alpha \cdot r)}(1+O(1 / \sqrt{k})),
$$

where the estimate of the remainder is uniform on any compact subset of $M$ and the remainder is bounded on $\bar{M}$, i.e, $\left|\frac{\partial}{\partial n} N(r)\right| \leq C k, \quad r \in M, \quad k \rightarrow \infty$. 
2. Let $K_{1}, K_{2}, K_{3}$ be compact sets strictly inside of the shadow zone or reflected zone, or strictly outside of these zones, respectively, and let $t_{0}$ be defined by (58). Then the following expansions are uniformly valid on these compact sets.

$$
\begin{array}{r}
N(r)=-2 \pi e^{i k(\alpha \cdot r)}+O(1 / \sqrt{k}), \quad r \in K_{1} \subset S(\alpha), \quad k \rightarrow \infty, \\
N(r)=2 \pi e^{i k t_{0}} e^{i k\left(\alpha^{*} \cdot r\right)}+O(1 / \sqrt{k}), \quad r \in K_{2} \subset R(\alpha), \quad k \rightarrow \infty, \\
N(r)=O(1 / \sqrt{k}), \quad r \in K_{3} \subset \mathbb{R}^{3} \backslash \overline{(R(\alpha) \cup S(\alpha))}, \quad k \rightarrow \infty
\end{array}
$$

These expansions on $K_{i}$ can be differentiated any number of times with the remainders multiplied by $k$ after each differentiation.

3. A uniform bound of $N(r)$ is valid in any bounded region:

$$
|N(r)|<C\left(R_{0}\right), \quad|\nabla N(r)|<k C\left(R_{0}\right), \quad|r|<R_{0}, r \notin \bar{M}, \quad k \rightarrow \infty .
$$

\section{References}

[1] Alena Aleksenko, Alexander Plakhov, Bodies of zero resistance and bodies invisible in one direction, Nonlinearity, 22, pp. 12471258, 2009.

[2] V. M. BABICH, On the asymptotics of Green's functions for certain wave problems. I. Stationary case, Mat. Sb. (N.S.), 86(128):4(12) (1971), pp. 518-537.

[3] Y. Cheng, F. Yang, J. Yi Xu, X. Jun LiU, A multilayer structured acoustic cloak with homogeneous isotropic materials, Appl. Phys. Lett. 92, 151913 (2008).

[4] E.L.Lakshtanov, Spectral properties of the Dirichlet-to-Neumann operator for the exterior Helmholtz problem and its applications to scattering theory, J. Phys. A: Math. Theor. 43 125204, 2009.

[5] E. Lakshtanov, B. VAinberg, Resonance regimes of scattering by small bodies with impedance boundary conditions, J. Phys. A: Math. Theor. 43 (2010) 415205 (16pp).

[6] D. Ludwig, Uniform asymptotic expansion of the field scattered by a convex object at high frequencies, Comm. pure and appl. math., 20, pp. 103138, (1967). 
[7] A. MAJDA, High frequency Asymptotics for the Scattering matrix and the inverse problem of Acoustical scattering, Comm. pure and applied math., vol. 29, pp. 261-291, (1976)

[8] A. Majda, M.E.TAYLOR, The asymptotic behavior of the diffractive peak in classical scattering, Comm. pure and applied math., vol. 30, pp. 639-669, (1977)

[9] W. MCLean, Strongly elliptic systems and boundary integral equations, Cambridge University Press, Cambridge, 2000.

[10] R. B. Melrose, Forward scattering by a convex obstacle, Comm Pure Appl. Math., 33, No 4 (1980), pp. 461-499.

[11] C. S. Morawetz, D. LudwiG, An inequality for reduced wave operator and the justification of geometrical optics, Comm. pure and appl. math., XXI, 2 (1968), pp. 111-118.

[12] A.N. Norris, Acoustic cloaking theory, Proc. R. Soc. A (2008) 464, pp. 2411-2434.

[13] V. PetKov, High frequency asymptotics of the scattering amplitude for non-convex bodies, Comm. Part. Diff. Equations, 5(1980), pp. 293-329.

[14] M. Schoenberg, P. N. Sen, Properties of a periodically stratified acoustic half-space and its relation to a Biot fluid J. Acoust. Soc. Am. Volume 73, Issue 1, pp. 61-67, (1986).

[15] B. R. VAInBERG, Quasi-classical approximation in stationary scattering problems, Funct. Analysis, Appl., 11 (1977), 247-257. 